\title{
Strong convergence theorem for a
} common fixed point of a finite family of strictly pseudo-contractive mappings and a strictly pseudononspreading mapping

Yifen Ke and Changfeng Ma*

${ }^{*}$ Correspondence: macf@fjnu.edu.cn School of Mathematics and Computer Science, Fujian Normal University, Fuzhou, 350117 P.R. China

\begin{abstract}
In this paper, we introduce a new mapping in a real Hilbert space to prove a strong convergence theorem for finding a common fixed point of a finite family of strictly pseudo-contractive mappings and a strictly pseudononspreading mapping. Moreover, we also obtain a strong convergence theorem for a finite family of inverse-strongly monotone mappings and a strictly pseudononspreading mapping.

MSC: 47H09; 47H10;49J40

Keywords: strictly pseudo-contractive mapping; strictly pseudononspreading mapping; inverse-strongly monotone mapping; strong convergence
\end{abstract}

\section{Introduction}

In this paper, we assume that $H$ is a real Hilbert space with the inner product $\langle\cdot, \cdot\rangle$ and the induced norm $\|\cdot\|$, and $C$ is a nonempty closed convex subset of $H$. Let $T: C \rightarrow C$ be a mapping. $F(T)$ denotes the set of fixed points of the mapping $T$, i.e., $F(T)=\{x \in C: T x=$ $x$.

Recall that a mapping $T: C \rightarrow C$ is nonexpansive if

$$
\|T x-T y\| \leq\|x-y\|, \quad \forall x, y \in C .
$$

A mapping $T: C \rightarrow C$ is $\kappa$-strictly pseudo-contractive if there exists a constant $\kappa \in[0,1)$ such that

$$
\|T x-T y\|^{2} \leq\|x-y\|^{2}+\kappa\|(I-T) x-(I-T) y\|^{2}, \quad \forall x, y \in C .
$$

A mapping $T: C \rightarrow C$ is $\rho$-strictly pseudononspreading if there exists a constant $\rho \in[0,1)$ such that

$$
\|T x-T y\|^{2} \leq\|x-y\|^{2}+\rho\|(I-T) x-(I-T) y\|^{2}+2\langle x-T x, y-T y\rangle, \quad \forall x, y \in C .
$$
(http://creativecommons.org/licenses/by/4.0/), which permits unrestricted use, distribution, and reproduction in any medium, provided you give appropriate credit to the original author(s) and the source, provide a link to the Creative Commons license, and indicate if changes were made. 
It is obvious that the 0 -strictly pseudo-contractive mapping $T$ is a nonexpansive mapping. Note that (1.2) is equivalent to

$$
\langle T x-T y, x-y\rangle \leq\|x-y\|^{2}-\frac{1-\kappa}{2}\|(I-T) x-(I-T) y\|^{2}, \quad \forall x, y \in C,
$$

and the $\kappa$-strictly pseudo-contractive mapping $T$ is Lipschitz continuous with constant $\frac{1+\kappa}{1-\kappa}$, that is,

$$
\|T x-T y\| \leq \frac{1+\kappa}{1-\kappa}\|x-y\|, \quad \forall x, y \in C .
$$

A mapping $T: C \rightarrow H$ is said to be $\xi$-inverse-strongly monotone if there exists a positive real number $\xi$ such that

$$
\langle T x-T y, x-y\rangle \geq \xi\|T x-T y\|^{2}, \quad \forall x, y \in C .
$$

Finding the fixed points of nonexpansive mappings is an important topic in the theory of nonexpansive mappings, and it has wide applications in a number of applied areas such as the convex feasibility problem [1-3], the split feasibility problem [4], image recovery and signal processing [5]. After that, as an important generalization of nonexpansive mappings, strictly pseudo-contractive, strictly pseudononspreading and inverse-strongly monotone mappings became one of the most interesting studied classes of nonexpansive mappings. Iterative methods for them have been extensively investigated (see, e.g., [6-19] and the references contained therein).

In 2000, Takahashi and Shimoji [20] introduced a $W$-mapping generated by $T_{1}, T_{2}$, $\ldots, T_{r}$ and $\alpha_{1}, \alpha_{2}, \ldots, \alpha_{r}$ as follows.

Definition 1.1 [20] Let $C$ be a convex subset of a Banach space $E$. Let $T_{1}, T_{2}, \ldots, T_{r}$ be finite mappings of $C$ into itself, and let $\alpha_{1}, \alpha_{2}, \ldots, \alpha_{r}$ be real numbers such that $0 \leq \alpha_{i} \leq 1$ for every $i=1,2, \ldots, r$. Then we define a mapping $W$ of $C$ into itself as follows:

$$
\begin{aligned}
& U_{1}=\alpha_{1} T_{1}+\left(1-\alpha_{1}\right) I, \\
& U_{2}=\alpha_{2} T_{2} U_{1}+\left(1-\alpha_{2}\right) I, \\
& U_{3}=\alpha_{3} T_{3} U_{2}+\left(1-\alpha_{3}\right) I, \\
& \vdots \\
& U_{r-1}=\alpha_{r-1} T_{r-1} U_{r-2}+\left(1-\alpha_{r-1}\right) I, \\
& W=U_{r}=\alpha_{r} T_{r} U_{r-1}+\left(1-\alpha_{r}\right) I .
\end{aligned}
$$

Such a mapping $W$ is called the $W$-mapping generated by $T_{1}, T_{2}, \ldots, T_{r}$ and $\alpha_{1}, \alpha_{2}, \ldots, \alpha_{r}$.

Lemma 1.1 [20] Let $C$ be a closed convex subset of a Banach space E. Let $T_{1}, T_{2}, \ldots, T_{r}$ be nonexpansive mappings of $C$ into itself such that $\bigcap_{i=1}^{r} F\left(T_{i}\right)$ is nonempty, and let $\alpha_{1}, \alpha_{2}, \ldots, \alpha_{r}$ be real numbers such that $0<\alpha_{i}<1$ for every $i=1,2, \ldots, r$. Let $W$ be the $W$ mapping of $C$ into itself generated by $T_{1}, T_{2}, \ldots, T_{r}$ and $\alpha_{1}, \alpha_{2}, \ldots, \alpha_{r}$. Then $W$ is asymptotically regular. Further, if $E$ is strictly convex, then $F(W)=\bigcap_{i=1}^{r} F\left(T_{i}\right)$. 
In 2009, Kangtunyakarn and Suantai [21] gave a $K$-mapping generated by $T_{1}, T_{2}, \ldots, T_{N}$ and $\lambda_{1}, \lambda_{2}, \ldots, \lambda_{N}$ as follows.

Definition 1.2 [21] Let $C$ be a nonempty convex subset of a real Banach space. Let $\left\{T_{i}\right\}_{i=1}^{N}$ be a finite family of mappings of $C$ into itself, and let $\lambda_{1}, \lambda_{2}, \ldots, \lambda_{N}$ be real numbers such that $0 \leq \lambda_{i} \leq 1$ for every $i=1,2, \ldots, N$. We define a mapping $K: C \rightarrow C$ as follows:

$$
\begin{aligned}
& U_{1}=\lambda_{1} T_{1}+\left(1-\lambda_{1}\right) I, \\
& U_{2}=\lambda_{2} T_{2} U_{1}+\left(1-\lambda_{2}\right) U_{1}, \\
& U_{3}=\lambda_{3} T_{3} U_{2}+\left(1-\lambda_{3}\right) U_{2}, \\
& \vdots \\
& U_{N-1}=\lambda_{N-1} T_{N-1} U_{N-2}+\left(1-\lambda_{N-1}\right) U_{N-2}, \\
& K=U_{N}=\lambda_{N} T_{N} U_{N-1}+\left(1-\lambda_{N}\right) U_{N-1} .
\end{aligned}
$$

Such a mapping $K$ is called the $K$-mapping generated by $T_{1}, T_{2}, \ldots, T_{N}$ and $\lambda_{1}, \lambda_{2}, \ldots, \lambda_{N}$.

In 2014, Suwannaut and Kangtunyakarn [22] established the following main result for the $K$-mapping generated by $T_{1}, T_{2}, \ldots T_{N}$ and $\lambda_{1}, \lambda_{2}, \ldots, \lambda_{N}$.

Lemma 1.2 [22] Let C be a nonempty closed convex subset of a real Hilbert space H. Let $\left\{T_{i}\right\}_{i=1}^{N}$ be a finite family of $\kappa_{i}$-strictly pseudo-contractive mappings of C into itself with $\kappa_{i} \leq$ $\gamma_{1}$ for all $i=1,2, \ldots, N$, and $\bigcap_{i=1}^{N} F\left(T_{i}\right) \neq \emptyset$. Let $\lambda_{1}, \lambda_{2}, \ldots, \lambda_{N}$ be real numbers with $0<\lambda_{i}<\gamma_{2}$ for all $i=1,2, \ldots, N$ and $\gamma_{1}+\gamma_{2}<1$. Let $K$ be the $K$-mapping generated by $T_{1}, T_{2}, \ldots, T_{N}$ and $\lambda_{1}, \lambda_{2}, \ldots, \lambda_{N}$. Then the following properties hold:

(i) $F(K)=\bigcap_{i=1}^{N} F\left(T_{i}\right)$;

(ii) $K$ is a nonexpansive mapping.

In 2009, Kangtunyakarn and Suantai [23] also introduced an $S$-mapping generated by $T_{1}, T_{2}, \ldots, T_{N}$ and $\alpha_{1}, \alpha_{2}, \ldots, \alpha_{N}$ as follows.

Definition 1.3 [23] Let $C$ be a nonempty convex subset of a real Banach space. Let $\left\{T_{i}\right\}_{i=1}^{N}$ be a finite family of mappings of $C$ into itself. For each $j=1,2, \ldots, N$, let $\alpha_{j}=\left(\alpha_{1}^{j}, \alpha_{2}^{j}, \alpha_{3}^{j}\right)$, where $\alpha_{1}^{j}, \alpha_{2}^{j}, \alpha_{3}^{j} \in[0,1]$ and $\alpha_{1}^{j}+\alpha_{2}^{j}+\alpha_{3}^{j}=1$. We define the mapping $S: C \rightarrow C$ as follows:

$$
\begin{aligned}
& U_{0}=I, \\
& U_{1}=\alpha_{1}^{1} T_{1} U_{0}+\alpha_{2}^{1} U_{0}+\alpha_{3}^{1} I, \\
& U_{2}=\alpha_{1}^{2} T_{2} U_{1}+\alpha_{2}^{2} U_{1}+\alpha_{3}^{2} I, \\
& U_{3}=\alpha_{1}^{3} T_{3} U_{2}+\alpha_{2}^{3} U_{2}+\alpha_{3}^{3} I, \\
& \vdots \\
& U_{N-1}=\alpha_{1}^{N-1} T_{N-1} U_{N-2}+\alpha_{2}^{N-1} U_{N-2}+\alpha_{3}^{N-1} I, \\
& S=U_{N}=\alpha_{1}^{N} T_{N} U_{N-1}+\alpha_{2}^{N} U_{N-1}+\alpha_{3}^{N} I .
\end{aligned}
$$

This mapping is called the $S$-mapping generated by $T_{1}, T_{2}, \ldots, T_{N}$ and $\alpha_{1}, \alpha_{2}, \ldots, \alpha_{N}$. 
In 2010, Kangtunyakarn and Suantai [24] gave the following lemma for the $S$-mapping generated by $T_{1}, T_{2}, \ldots, T_{N}$ and $\alpha_{1}, \alpha_{2}, \ldots, \alpha_{N}$.

Lemma 1.3 [24] Let $C$ be a nonempty closed convex subset of a real Hilbert space. Let $\left\{T_{i}\right\}_{i=1}^{N}$ be a finite family of $\kappa_{i}$-strict pseudocontractive mappings of $C$ into $C$ with $\bigcap_{i=1}^{N} F\left(T_{i}\right) \neq \emptyset$ and $\kappa=\max \left\{\kappa_{i}: i=1,2, \ldots, N\right\}$, and let $\alpha_{j}=\left(\alpha_{1}^{j}, \alpha_{2}^{j}, \alpha_{3}^{j}\right) \in I \times I \times I, j=$ $1,2, \ldots, N$, where $I=[0,1], \alpha_{1}^{j}+\alpha_{2}^{j}+\alpha_{3}^{j}=1, \alpha_{1}^{j}, \alpha_{3}^{j} \in(\kappa, 1)$ for all $j=1,2, \ldots, N-1$ and $\alpha_{1}^{N} \in(\kappa, 1], \alpha_{3}^{N} \in[\kappa, 1), \alpha_{2}^{j} \in[\kappa, 1)$ for all $j=1,2, \ldots, N$. Let $S$ be the mapping generated by $T_{1}, T_{2}, \ldots, T_{N}$ and $\alpha_{1}, \alpha_{2}, \ldots, \alpha_{N}$. Then $F(S)=\bigcap_{i=1}^{N} F\left(T_{i}\right)$ and $S$ is a nonexpansive mapping.

Let $T: C \rightarrow H$. The variational inequality problem is to find a point $x \in C$ such that

$$
\langle A x, y-x\rangle \geq 0, \quad \forall y \in C
$$

The set of solutions of (1.7) is denoted by $V I(C, A)$.

In the recent years, there have been many research works concerning the problem of approximating a common fixed point of various classes of nonlinear mappings by using $W$-mappings, $K$-mappings and $S$-mappings (see, e.g., [20-43]).

Recently, Kangtunyakarn [44] proposed an iterative algorithm for finding a common element of the set of fixed points of a $\kappa$-strictly pseudononspreading mapping and a finite family of the set of solutions of variational inequality problems as follows.

Theorem 1.1 [44] Let $H$ be a real Hilbert space and let $C$ be a nonempty closed convex subset of $H$. For every $i=1,2, \ldots, N$, let $B_{i}: C \rightarrow H$ be $\delta_{i}$-inverse strongly monotone mappings and let $T: C \rightarrow C$ be a $\kappa$-strictly pseudononspreading mapping for some $\kappa \in[0,1)$. Let $G_{i}: C \rightarrow C$ be defined by $G_{i} x=P_{C}\left(I-\eta B_{i}\right) x$ for every $x \in C$ and $\eta \in\left(0,2 \delta_{i}\right)$ for every $i=1,2, \ldots, N$, and let $\delta_{j}=\left(\alpha_{1}^{j}, \alpha_{2}^{j}, \alpha_{3}^{j}\right) \in I \times I \times I, j=1,2, \ldots, N$, where $I=[0,1]$, $\alpha_{1}^{j}+\alpha_{2}^{j}+\alpha_{3}^{j}=1, \alpha_{1}^{j} \in(0,1)$ for all $j=1,2, \ldots, N-1, \alpha_{1}^{N} \in(0,1], \alpha_{2}^{j}, \alpha_{3}^{j} \in[0,1)$ for all $j=1,2, \ldots, N$. Let $S: C \rightarrow C$ be the $S$-mapping generated by $G_{1}, G_{2}, \ldots, G_{N}$ and $\delta_{1}, \delta_{2}, \ldots, \delta_{N}$. Assume that $\mathfrak{F}=F(T) \cap \bigcap_{i=1}^{N} V I\left(C, B_{i}\right) \neq \emptyset$. For every $n \in \mathbb{N}, i=1,2, \ldots, N$, let $x_{1}, u \in C$ and $\left\{x_{n}\right\}$ be a sequence generated by

$$
x_{n+1}=\alpha_{n} u+\beta_{n} P_{C}\left(I-\lambda_{n}(I-T)\right) x_{n}+\gamma_{n} S x_{n}, \quad \forall n \in \mathbb{N},
$$

where $\left\{\alpha_{n}\right\},\left\{\beta_{n}\right\},\left\{\gamma_{n}\right\},\left\{\lambda_{n}\right\} \subset(0,1)$ such that $\alpha_{n}+\beta_{n}+\gamma_{n}=1, \beta_{n} \in[c, d] \subset(0,1),\left\{\lambda_{n}\right\} \subset$ $(0,1-\kappa)$ and suppose the following conditions hold:

(i) $\lim _{n \rightarrow \infty} \alpha_{n}=0$ and $\sum_{n=0}^{\infty} \alpha_{n}=\infty$;

(ii) $\sum_{n=1}^{\infty} \lambda_{n}<\infty$;

(iii) $\sum_{n=1}^{\infty}\left|\lambda_{n+1}-\lambda_{n}\right|, \sum_{n=1}^{\infty}\left|\gamma_{n+1}-\gamma_{n}\right|, \sum_{n=1}^{\infty}\left|\alpha_{n+1}-\alpha_{n}\right|, \sum_{n=1}^{\infty}\left|\beta_{n+1}-\beta_{n}\right|<\infty$.

Then the sequence $\left\{x_{n}\right\}$ converges strongly to $z=P_{\mathfrak{F}} u$.

Motivated and inspired by the above facts, we define a new mapping for the common fixed point set of a finite family of strict pseudo-contractive mappings. Moreover, by using our main result, we also obtain a new strong convergence theorem for the common fixed point of a finite family of strict pseudo-contractive mappings and a strictly pseudononspreading mapping. 


\section{Preliminaries}

Lemma 2.1 In the real Hilbert space $H$, the following relations hold:

(i) $\|x+y\|^{2}=\|x\|^{2}+2\langle x, y\rangle+\|y\|^{2}$;

(ii) $\|x+y\|^{2} \leq\|x\|^{2}+2\langle y, x+y\rangle$;

(iii) $\left\|\sum_{i=1}^{m} \alpha_{i} x_{i}\right\|^{2}=\sum_{i=1}^{m} \alpha_{i}\left\|x_{i}\right\|^{2}-\sum_{i \neq j} \alpha_{i} \alpha_{j}\left\|x_{i}-x_{j}\right\|^{2}$

for $\sum_{i=1}^{m} \alpha_{i}=1, \alpha_{i} \in[0,1], \forall i \in\{1,2, \ldots, m\}$.

Definition 2.1 $P_{C}: H \rightarrow C$ is called a metric projection if for every point $x \in H$, there exists a unique nearest point in $C$, denoted by $P_{C} x$, such that

$$
\left\|x-P_{C} x\right\| \leq\|x-y\|, \quad \forall y \in C .
$$

Lemma 2.2 Let $C$ be a nonempty closed convex subset of $H$ and $P_{C}: H \rightarrow C$ be a metric projection. Then

(i) $\left\|P_{C} x-P_{C} y\right\|^{2} \leq\left\langle x-y, P_{C} x-P_{C} y\right\rangle, \forall x, y \in H$;

(ii) $P_{C}$ is a nonexpansive mapping, i.e., $\left\|P_{C} x-P_{C} y\right\| \leq\|x-y\|, \forall x, y \in H$;

(iii) $\left\langle x-P_{C} x, y-P_{C} x\right\rangle \leq 0, \forall x \in H, y \in C$.

From the proof of Theorem 3.1 in [44], we have the following results.

Lemma 2.3 [44] Let $C$ be a nonempty closed convex subset of $H$ and $T: C \rightarrow C$ be a $\rho$ strictly pseudononspreading mapping with $F(T) \neq \emptyset$. Then

$$
\left\|P_{C}(I-\lambda(I-T)) x-x^{*}\right\| \leq\left\|x-x^{*}\right\|
$$

for any $\lambda \in(0,1-\rho), x^{*} \in F(T)$.

Lemma 2.4 [44] Let $C$ be a nonempty closed convex subset of $H$ and $T: C \rightarrow C$ be a $\rho$ strictly pseudononspreading mapping with $F(T) \neq \emptyset$. Then

$$
\left\|T x-x^{*}\right\| \leq \frac{1+\rho}{1-\rho}\left\|x-x^{*}\right\|
$$

for any $x^{*} \in F(T)$.

Lemma 2.5 [45] Let $\left\{s_{n}\right\}$ be a sequence of nonnegative real numbers such that

$$
s_{n+1} \leq\left(1-\alpha_{n}\right) s_{n}+\beta_{n}, \quad \forall n \geq 0,
$$

where $\left\{\alpha_{n}\right\}$ is a sequence in $(0,1)$ and $\left\{\beta_{n}\right\}$ is a sequence such that

(i) $\sum_{n=0}^{\infty} \alpha_{n}=\infty$;

(ii) $\lim \sup _{n \rightarrow \infty} \frac{\beta_{n}}{\alpha_{n}} \leq 0$ or $\sum_{n=0}^{\infty}\left|\beta_{n}\right|<\infty$.

Then $\lim _{n \rightarrow \infty} s_{n}=0$.

Lemma 2.6 [45] Let $\left\{s_{n}\right\}$ be a sequence of nonnegative numbers such that

$$
s_{n+1} \leq\left(1-\alpha_{n}\right) s_{n}+\alpha_{n} \beta_{n}, \quad \forall n \geq 0,
$$

where $\left\{\alpha_{n}\right\}$ and $\left\{\beta_{n}\right\}$ are sequences of real numbers such that

(i) $\left\{\alpha_{n}\right\} \subset[0,1]$ and $\sum_{n=0}^{\infty} \alpha_{n}=\infty$; 
(ii) $\lim \sup _{n \rightarrow \infty} \beta_{n} \leq 0$ or $\sum_{n=0}^{\infty} \alpha_{n} \beta_{n}<\infty$.

Then $\lim _{n \rightarrow \infty} s_{n}=0$.

Let $C$ be a nonempty subset of $H$ and $T: C \rightarrow H$ be a mapping. Then $T$ is said to be demi-closed at $v \in H$ if for any sequence $\left\{x_{n}\right\} \subseteq C$, the following implication holds:

$$
x_{n} \rightarrow u \in C \text { and } T x_{n} \rightarrow v \text { imply } T u=v \text {, }
$$

where $\rightarrow$ (resp. $\rightarrow$ ) denotes strong (resp. weak) convergence.

Lemma 2.7 [46] Let $C$ be a nonempty closed convex subset of $H$ and $T: C \rightarrow H$ be a nonexpansive mapping. Then the mapping $I-T$ is demi-closed at zero.

Lemma 2.8 (Opial's property [47]) If $x_{n} \rightarrow u$, then the following inequality holds:

$$
\liminf _{n \rightarrow \infty}\left\|x_{n}-y\right\|>\liminf _{n \rightarrow \infty}\left\|x_{n}-u\right\|, \quad \forall y \in H, y \neq u
$$

We define a new mapping as follows.

Definition 2.2 Let $C$ be a nonempty convex subset of a Banach space $E$. Let $\left\{T_{i}\right\}_{i=1}^{N}$ be a finite family of mappings of $C$ into itself. For each $i=1,2, \ldots, N$, let $\pi_{i}=\left(\alpha_{i}, \beta_{i}, \gamma_{i}, \delta_{i}\right)$, where $\alpha_{i}, \beta_{i}, \gamma_{i}, \delta_{i} \in[0,1]$ and $\alpha_{i}+\beta_{i}+\gamma_{i}+\delta_{i}=1$. We define the mapping $G: C \rightarrow C$ as follows:

$$
\begin{aligned}
& U_{0}=I, \\
& U_{1}=\alpha_{1} T_{1}^{2} U_{0}+\beta_{1} T_{1} U_{0}+\gamma_{1} U_{0}+\delta_{1} I, \\
& U_{2}=\alpha_{2} T_{2}^{2} U_{1}+\beta_{2} T_{2} U_{1}+\gamma_{2} U_{1}+\delta_{2} I, \\
& U_{3}=\alpha_{3} T_{3}^{2} U_{2}+\beta_{3} T_{3} U_{2}+\gamma_{3} U_{2}+\delta_{3} I, \\
& \vdots \\
& U_{N-1}=\alpha_{N-1} T_{N-1}^{2} U_{N-2}+\beta_{N-1} T_{N-1} U_{N-2}+\gamma_{N-1} U_{N-2}+\delta_{N-1} I, \\
& G=U_{N}=\alpha_{N} T_{N}^{2} U_{N-1}+\beta_{N} T_{N} U_{N-1}+\gamma_{N} U_{N-1}+\delta_{N} I .
\end{aligned}
$$

This mapping is called the $G$-mapping generated by $T_{1}, T_{2}, \ldots, T_{N}$ and $\pi_{1}, \pi_{2}, \ldots, \pi_{N}$.

We remark that (i) if $\alpha_{i}=0$ for every $i=1,2, \ldots, N$, then $G$-mapping is reduced to $S$ mapping; (ii) if $\alpha_{i}=0$ and $\gamma_{i}=0$ for every $i=1,2, \ldots, N$, then $G$-mapping is reduced to $W$-mapping; (iii) if $\alpha_{i}=0$ and $\delta_{i}=0$ for every $i=1,2, \ldots, N$, then $G$-mapping is reduced to $K$-mapping.

Lemma 2.9 Let $C$ be a nonempty closed convex subset of the real Hilbert space $H$. For every $i=1,2, \ldots, N$, let $T_{i}: C \rightarrow C$ be $\kappa_{i}$-strict pseudo-contractive mappings with $\bigcap_{i=1}^{N} F\left(T_{i}\right) \neq \emptyset$, and let $\pi_{i}=\left(\alpha_{i}, \beta_{i}, \gamma_{i}, \delta_{i}\right)$, where $\alpha_{i}, \beta_{i}, \gamma_{i}, \delta_{i} \in[0,1]$ and $\alpha_{i}+\beta_{i}+\gamma_{i}+\delta_{i}=1$. Let $G$ be the G-mapping generated by $T_{1}, T_{2}, \ldots, T_{N}$ and $\pi_{1}, \pi_{2}, \ldots, \pi_{N}$. If the following conditions hold:

(i) $\kappa_{1} \leq \beta_{1}<1-\kappa_{1}$ and $\alpha_{1}\left(\kappa_{1}+\beta_{1}\right)<\beta_{1}\left(1-\beta_{1}-\kappa_{1}\right)$;

(ii) $\beta_{i} \geq \kappa_{i}, \kappa_{i}<\gamma_{i}<1$ and $\kappa_{i} \alpha_{i} \leq \beta_{i} \gamma_{i}-\beta_{i} \kappa_{i}$ for $i=2,3, \ldots, N$.

Then $F(G)=\bigcap_{i=1}^{N} F\left(T_{i}\right)$ and $G$ is a nonexpansive mapping. 
Proof It is clear that $\bigcap_{i=1}^{N} F\left(T_{i}\right) \subseteq F(G)$. Next, we will show that $F(G) \subseteq \bigcap_{i=1}^{N} F\left(T_{i}\right)$. Let $x_{0} \in F(G)$ and $x^{*} \in \bigcap_{i=1}^{N} F\left(T_{i}\right)$, then we have

$$
\begin{aligned}
& \left\|x_{0}-x^{*}\right\|^{2} \\
& =\left\|G x_{0}-x^{*}\right\|^{2} \\
& =\left\|\alpha_{N}\left(T_{N}^{2} U_{N-1} x_{0}-x^{*}\right)+\beta_{N}\left(T_{N} U_{N-1} x_{0}-x^{*}\right)+\gamma_{N}\left(U_{N-1} x_{0}-x^{*}\right)+\delta_{N}\left(x_{0}-x^{*}\right)\right\|^{2} \\
& =\alpha_{N}\left\|T_{N}^{2} U_{N-1} x_{0}-x^{*}\right\|^{2}+\beta_{N}\left\|T_{N} U_{N-1} x_{0}-x^{*}\right\|^{2} \\
& +\gamma_{N}\left\|U_{N-1} x_{0}-x^{*}\right\|^{2}+\delta_{N}\left\|x_{0}-x^{*}\right\|^{2} \\
& -\alpha_{N} \beta_{N}\left\|T_{N}^{2} U_{N-1} x_{0}-T_{N} U_{N-1} x_{0}\right\|^{2}-\alpha_{N} \gamma_{N}\left\|T_{N}^{2} U_{N-1} x_{0}-U_{N-1} x_{0}\right\|^{2} \\
& -\alpha_{N} \delta_{N}\left\|T_{N}^{2} U_{N-1} x_{0}-x_{0}\right\|^{2}-\beta_{N} \gamma_{N}\left\|T_{N} U_{N-1} x_{0}-U_{N-1} x_{0}\right\|^{2} \\
& -\beta_{N} \delta_{N}\left\|T_{N} U_{N-1} x_{0}-x_{0}\right\|^{2}-\gamma_{N} \delta_{N}\left\|U_{N-1} x_{0}-x_{0}\right\|^{2} \\
& \leq \alpha_{N}\left\|T_{N}^{2} U_{N-1} x_{0}-x^{*}\right\|^{2}+\beta_{N}\left\|T_{N} U_{N-1} x_{0}-x^{*}\right\|^{2} \\
& +\gamma_{N}\left\|U_{N-1} x_{0}-x^{*}\right\|^{2}+\delta_{N}\left\|x_{0}-x^{*}\right\|^{2}-\alpha_{N} \beta_{N}\left\|T_{N}^{2} U_{N-1} x_{0}-T_{N} U_{N-1} x_{0}\right\|^{2} \\
& -\beta_{N} \gamma_{N}\left\|T_{N} U_{N-1} x_{0}-U_{N-1} x_{0}\right\|^{2} \\
& \leq \alpha_{N}\left(\left\|T_{N} U_{N-1} x_{0}-x^{*}\right\|^{2}+\kappa_{N}\left\|\left(I-T_{N}\right) T_{N} U_{N-1} x_{0}\right\|^{2}\right) \\
& +\beta_{N}\left\|T_{N} U_{N-1} x_{0}-x^{*}\right\|^{2}+\gamma_{N}\left\|U_{N-1} x_{0}-x^{*}\right\|^{2}+\delta_{N}\left\|x_{0}-x^{*}\right\|^{2} \\
& -\alpha_{N} \beta_{N}\left\|T_{N}^{2} U_{N-1} x_{0}-T_{N} U_{N-1} x_{0}\right\|^{2}-\beta_{N} \gamma_{N}\left\|T_{N} U_{N-1} x_{0}-U_{N-1} x_{0}\right\|^{2} \\
& =\left(\alpha_{N}+\beta_{N}\right)\left\|T_{N} U_{N-1} x_{0}-x^{*}\right\|^{2}+\alpha_{N}\left(\kappa_{N}-\beta_{N}\right)\left\|T_{N}^{2} U_{N-1} x_{0}-T_{N} U_{N-1} x_{0}\right\|^{2} \\
& +\gamma_{N}\left\|U_{N-1} x_{0}-x^{*}\right\|^{2}+\delta_{N}\left\|x_{0}-x^{*}\right\|^{2}-\beta_{N} \gamma_{N}\left\|T_{N} U_{N-1} x_{0}-U_{N-1} x_{0}\right\|^{2} \\
& \leq\left(\alpha_{N}+\beta_{N}\right)\left(\left\|U_{N-1} x_{0}-x^{*}\right\|^{2}+\kappa_{N}\left\|\left(I-T_{N}\right) U_{N-1} x_{0}\right\|^{2}\right) \\
& +\alpha_{N}\left(\kappa_{N}-\beta_{N}\right)\left\|T_{N}^{2} U_{N-1} x_{0}-T_{N} U_{N-1} x_{0}\right\|^{2} \\
& +\gamma_{N}\left\|U_{N-1} x_{0}-x^{*}\right\|^{2}+\delta_{N}\left\|x_{0}-x^{*}\right\|^{2}-\beta_{N} \gamma_{N}\left\|T_{N} U_{N-1} x_{0}-U_{N-1} x_{0}\right\|^{2} \\
& =\left(1-\delta_{N}\right)\left\|U_{N-1} x_{0}-x^{*}\right\|^{2}+\left(1-\left(1-\delta_{N}\right)\right)\left\|x_{0}-x^{*}\right\|^{2} \\
& +\alpha_{N}\left(\kappa_{N}-\beta_{N}\right)\left\|T_{N}^{2} U_{N-1} x_{0}-T_{N} U_{N-1} x_{0}\right\|^{2} \\
& +\left(\left(\alpha_{N}+\beta_{N}\right) \kappa_{N}-\beta_{N} \gamma_{N}\right)\left\|T_{N} U_{N-1} x_{0}-U_{N-1} x_{0}\right\|^{2} \\
& \leq\left(1-\delta_{N}\right)\left\|U_{N-1} x_{0}-x^{*}\right\|^{2}+\left(1-\left(1-\delta_{N}\right)\right)\left\|x_{0}-x^{*}\right\|^{2} \\
& \vdots \\
& \leq\left(1-\delta_{N}\right)\left[\left(1-\delta_{N-1}\right)\left\|U_{N-2} x_{0}-x^{*}\right\|^{2}+\left(1-\left(1-\delta_{N-1}\right)\right)\left\|x_{0}-x^{*}\right\|^{2}\right] \\
& +\left(1-\left(1-\delta_{N}\right)\right)\left\|x_{0}-x^{*}\right\|^{2} \\
& =\left(1-\delta_{N}\right)\left(1-\delta_{N-1}\right)\left\|U_{N-2} x_{0}-x^{*}\right\|^{2}+\left(1-\left(1-\delta_{N}\right)\left(1-\delta_{N-1}\right)\right)\left\|x_{0}-x^{*}\right\|^{2}
\end{aligned}
$$


Xe and Ma Fixed Point Theory and Applications ( 2015) 2015:116

Page 8 of 23

$$
\begin{aligned}
& \leq \prod_{i=3}^{N}\left(1-\delta_{i}\right)\left\|U_{2} x_{0}-x^{*}\right\|^{2}+\left(1-\prod_{i=3}^{N}\left(1-\delta_{i}\right)\right)\left\|x_{0}-x^{*}\right\|^{2} \\
& \leq \prod_{i=3}^{N}\left(1-\delta_{i}\right)\left[\left(1-\delta_{2}\right)\left\|U_{1} x_{0}-x^{*}\right\|^{2}+\delta_{2}\left\|x_{0}-x^{*}\right\|^{2}\right. \\
& \left.+\alpha_{2}\left(\kappa_{2}-\beta_{2}\right)\left\|T_{2}^{2} U_{1} x_{0}-T_{2} U_{1} x_{0}\right\|^{2}+\left(\left(\alpha_{2}+\beta_{2}\right) \kappa_{2}-\beta_{2} \gamma_{2}\right)\left\|T_{2} U_{1} x_{0}-U_{1} x_{0}\right\|^{2}\right] \\
& +\left(1-\prod_{i=3}^{N}\left(1-\delta_{i}\right)\right)\left\|x_{0}-x^{*}\right\|^{2} \\
& \leq \prod_{i=2}^{N}\left(1-\delta_{i}\right)\left\|U_{1} x_{0}-x^{*}\right\|^{2}+\left(1-\prod_{i=2}^{N}\left(1-\delta_{i}\right)\right)\left\|x_{0}-x^{*}\right\|^{2} \\
& =\prod_{i=2}^{N}\left(1-\delta_{i}\right)\left\|\alpha_{1}\left(T_{1}^{2} x_{0}-x^{*}\right)+\beta_{1}\left(T_{1} x_{0}-x^{*}\right)+\left(1-\alpha_{1}-\beta_{1}\right)\left(x_{0}-x^{*}\right)\right\|^{2} \\
& +\left(1-\prod_{i=2}^{N}\left(1-\delta_{i}\right)\right)\left\|x_{0}-x^{*}\right\|^{2} \\
& =\prod_{i=2}^{N}\left(1-\delta_{i}\right)\left[\alpha_{1}\left\|T_{1}^{2} x_{0}-x^{*}\right\|^{2}+\beta_{1}\left\|T_{1} x_{0}-x^{*}\right\|^{2}+\left(1-\alpha_{1}-\beta_{1}\right)\left\|x_{0}-x^{*}\right\|^{2}\right. \\
& -\alpha_{1} \beta_{1}\left\|T_{1}^{2} x_{0}-T_{1} x_{0}\right\|^{2}-\alpha_{1}\left(1-\alpha_{1}-\beta_{1}\right)\left\|T_{1}^{2} x_{0}-x_{0}\right\|^{2} \\
& \left.-\beta_{1}\left(1-\alpha_{1}-\beta_{1}\right)\left\|T_{1} x_{0}-x_{0}\right\|^{2}\right]+\left(1-\prod_{i=2}^{N}\left(1-\delta_{i}\right)\right)\left\|x_{0}-x^{*}\right\|^{2} \\
& \leq \prod_{i=2}^{N}\left(1-\delta_{i}\right)\left[\alpha_{1}\left\|T_{1}^{2} x_{0}-x^{*}\right\|^{2}+\beta_{1}\left\|T_{1} x_{0}-x^{*}\right\|^{2}+\left(1-\alpha_{1}-\beta_{1}\right)\left\|x_{0}-x^{*}\right\|^{2}\right. \\
& \left.-\alpha_{1} \beta_{1}\left\|T_{1}^{2} x_{0}-T_{1} x_{0}\right\|^{2}-\beta_{1}\left(1-\alpha_{1}-\beta_{1}\right)\left\|T_{1} x_{0}-x_{0}\right\|^{2}\right] \\
& +\left(1-\prod_{i=2}^{N}\left(1-\delta_{i}\right)\right)\left\|x_{0}-x^{*}\right\|^{2} \\
& \leq \prod_{i=2}^{N}\left(1-\delta_{i}\right)\left[\alpha_{1}\left(\left\|T_{1} x_{0}-x^{*}\right\|^{2}+\kappa_{1}\left\|\left(I-T_{1}\right) T_{1} x_{0}\right\|^{2}\right)+\beta_{1}\left\|T_{1} x_{0}-x^{*}\right\|^{2}\right. \\
& \left.+\left(1-\alpha_{1}-\beta_{1}\right)\left\|x_{0}-x^{*}\right\|^{2}-\alpha_{1} \beta_{1}\left\|T_{1}^{2} x_{0}-T_{1} x_{0}\right\|^{2}-\beta_{1}\left(1-\alpha_{1}-\beta_{1}\right)\left\|T_{1} x_{0}-x_{0}\right\|^{2}\right] \\
& +\left(1-\prod_{i=2}^{N}\left(1-\delta_{i}\right)\right)\left\|x_{0}-x^{*}\right\|^{2} \\
& =\prod_{i=2}^{N}\left(1-\delta_{i}\right)\left[\left(\alpha_{1}+\beta_{1}\right)\left\|T_{1} x_{0}-x^{*}\right\|^{2}+\alpha_{1}\left(\kappa_{1}-\beta_{1}\right)\left\|T_{1}^{2} x_{0}-T_{1} x_{0}\right\|^{2}\right. \\
& \left.+\left(1-\alpha_{1}-\beta_{1}\right)\left\|x_{0}-x^{*}\right\|^{2}-\beta_{1}\left(1-\alpha_{1}-\beta_{1}\right)\left\|T_{1} x_{0}-x_{0}\right\|^{2}\right] \\
& +\left(1-\prod_{i=2}^{N}\left(1-\delta_{i}\right)\right)\left\|x_{0}-x^{*}\right\|^{2}
\end{aligned}
$$




$$
\begin{aligned}
\leq & \prod_{i=2}^{N}\left(1-\delta_{i}\right)\left[\left(\alpha_{1}+\beta_{1}\right)\left(\left\|x_{0}-x^{*}\right\|^{2}+\kappa_{1}\left\|\left(I-T_{1}\right) x_{0}\right\|^{2}\right)+\alpha_{1}\left(\kappa_{1}-\beta_{1}\right)\left\|T_{1}^{2} x_{0}-T_{1} x_{0}\right\|^{2}\right. \\
& \left.+\left(1-\alpha_{1}-\beta_{1}\right)\left\|x_{0}-x^{*}\right\|^{2}-\beta_{1}\left(1-\alpha_{1}-\beta_{1}\right)\left\|T_{1} x_{0}-x_{0}\right\|^{2}\right] \\
& +\left(1-\prod_{i=2}^{N}\left(1-\delta_{i}\right)\right)\left\|x_{0}-x^{*}\right\|^{2} \\
= & \prod_{i=2}^{N}\left(1-\delta_{i}\right)\left[\left\|x_{0}-x^{*}\right\|^{2}+\alpha_{1}\left(\kappa_{1}-\beta_{1}\right)\left\|T_{1}^{2} x_{0}-T_{1} x_{0}\right\|^{2}\right. \\
& \left.+\left(\left(\alpha_{1}+\beta_{1}\right) \kappa_{1}-\beta_{1}\left(1-\alpha_{1}-\beta_{1}\right)\right)\left\|T_{1} x_{0}-x_{0}\right\|^{2}\right] \\
& +\left(1-\prod_{i=2}^{N}\left(1-\delta_{i}\right)\right)\left\|x_{0}-x^{*}\right\|^{2} .
\end{aligned}
$$

By the condition (i), we have

$$
\alpha_{1}\left(\kappa_{1}-\beta_{1}\right)\left\|T_{1}^{2} x_{0}-T_{1} x_{0}\right\|^{2}+\left(\left(\alpha_{1}+\beta_{1}\right) \kappa_{1}-\beta_{1}\left(1-\alpha_{1}-\beta_{1}\right)\right)\left\|T_{1} x_{0}-x_{0}\right\|^{2} \leq 0 .
$$

From (2.9) and $\delta_{i}<1$ for $i=2,3, \ldots, N$, it yields

$$
\alpha_{1}\left(\kappa_{1}-\beta_{1}\right)\left\|T_{1}^{2} x_{0}-T_{1} x_{0}\right\|^{2}+\left(\left(\alpha_{1}+\beta_{1}\right) \kappa_{1}-\beta_{1}\left(1-\alpha_{1}-\beta_{1}\right)\right)\left\|T_{1} x_{0}-x_{0}\right\|^{2} \geq 0 .
$$

This implies that

$$
\left\|T_{1} x_{0}-x_{0}\right\|=0
$$

Therefore, $T_{1} x_{0}=x_{0}$, that is, $x_{0} \in F\left(T_{1}\right)$. By the definition of $U_{1}$, we have

$$
\begin{aligned}
U_{1} x_{0} & =\alpha_{1} T_{1}^{2} U_{0} x_{0}+\beta_{1} T_{1} U_{0} x_{0}+\gamma_{1} U_{0} x_{0}+\delta_{1} x_{0} \\
& =\alpha_{1} T_{1}^{2} x_{0}+\beta_{1} T_{1} x_{0}+\gamma_{1} x_{0}+\delta_{1} x_{0} \\
& =\alpha_{1} T_{1} x_{0}+\beta_{1} x_{0}+\gamma_{1} x_{0}+\delta_{1} x_{0} \\
& =x_{0} .
\end{aligned}
$$

Again, by (2.8), (2.13) and $\delta_{i}<1$ for $i=3,4, \ldots, N$, we have

$$
\begin{aligned}
& \alpha_{2}\left(\kappa_{2}-\beta_{2}\right)\left\|T_{2}^{2} U_{1} x_{0}-T_{2} U_{1} x_{0}\right\|^{2}+\left(\left(\alpha_{2}+\beta_{2}\right) \kappa_{2}-\beta_{2} \gamma_{2}\right)\left\|T_{2} U_{1} x_{0}-U_{1} x_{0}\right\|^{2} \\
& \quad=\alpha_{2}\left(\kappa_{2}-\beta_{2}\right)\left\|T_{2}^{2} x_{0}-T_{2} x_{0}\right\|^{2}+\left(\left(\alpha_{2}+\beta_{2}\right) \kappa_{2}-\beta_{2} \gamma_{2}\right)\left\|T_{2} x_{0}-x_{0}\right\|^{2} \\
& \quad \geq 0 .
\end{aligned}
$$

From the condition (ii), this implies

$$
\left\|T_{2} x_{0}-x_{0}\right\|=0
$$

Therefore, $T_{2} x_{0}=x_{0}$, that is, $x_{0} \in F\left(T_{2}\right)$. By the definition of $U_{2}$, we also have

$$
U_{2} x_{0}=x_{0} .
$$


Using the same argument, we can conclude that

$$
x_{0} \in F\left(T_{i}\right), \quad i=3,4, \ldots, N .
$$

Hence, $F(G) \subseteq \bigcap_{i=1}^{N} F\left(T_{i}\right)$.

Now, we show that $G$ is nonexpansive. Let any $x, y \in C$. Then

$$
\begin{aligned}
& \|G x-G y\|^{2} \\
& =\| \alpha_{N}\left(T_{N}^{2} U_{N-1} x-T_{N}^{2} U_{N-1} y\right)+\beta_{N}\left(T_{N} U_{N-1} x-T_{N} U_{N-1} y\right) \\
& +\gamma_{N}\left(U_{N-1} x-U_{N-1} y\right)+\delta_{N}(x-y) \|^{2} \\
& \leq \alpha_{N}\left\|T_{N}^{2} U_{N-1} x-T_{N}^{2} U_{N-1} y\right\|^{2}+\beta_{N}\left\|T_{N} U_{N-1} x-T_{N} U_{N-1} y\right\|^{2} \\
& +\gamma_{N}\left\|U_{N-1} x-U_{N-1} y\right\|^{2}+\delta_{N}\|x-y\|^{2} \\
& -\alpha_{N} \beta_{N}\left\|\left(I-T_{N}\right) T_{N} U_{N-1} x-\left(I-T_{N}\right) T_{N} U_{N-1} y\right\|^{2} \\
& -\beta_{N} \gamma_{N}\left\|\left(I-T_{N}\right) U_{N-1} x-\left(I-T_{N}\right) U_{N-1} y\right\|^{2} \\
& \leq \alpha_{N}\left(\left\|T_{N} U_{N-1} x-T_{N} U_{N-1} y\right\|^{2}+\kappa_{N}\left\|\left(I-T_{N}\right) T_{N} U_{N-1} x-\left(I-T_{N}\right) T_{N} U_{N-1} y\right\|^{2}\right) \\
& +\beta_{N}\left\|T_{N} U_{N-1} x-T_{N} U_{N-1} y\right\|^{2}+\gamma_{N}\left\|U_{N-1} x-U_{N-1} y\right\|^{2}+\delta_{N}\|x-y\|^{2} \\
& -\alpha_{N} \beta_{N}\left\|\left(I-T_{N}\right) T_{N} U_{N-1} x-\left(I-T_{N}\right) T_{N} U_{N-1} y\right\|^{2} \\
& -\beta_{N} \gamma_{N}\left\|\left(I-T_{N}\right) U_{N-1} x-\left(I-T_{N}\right) U_{N-1} y\right\|^{2} \\
& =\left(\alpha_{N}+\beta_{N}\right)\left\|T_{N} U_{N-1} x-T_{N} U_{N-1} y\right\|^{2} \\
& +\alpha_{N}\left(\kappa_{N}-\beta_{N}\right)\left\|\left(I-T_{N}\right) T_{N} U_{N-1} x-\left(I-T_{N}\right) T_{N} U_{N-1} y\right\|^{2} \\
& +\gamma_{N}\left\|U_{N-1} x-U_{N-1} y\right\|^{2}+\delta_{N}\|x-y\|^{2} \\
& -\beta_{N} \gamma_{N}\left\|\left(I-T_{N}\right) U_{N-1} x-\left(I-T_{N}\right) U_{N-1} y\right\|^{2} \\
& \leq\left(\alpha_{N}+\beta_{N}\right)\left(\left\|U_{N-1} x-U_{N-1} y\right\|^{2}+\kappa_{N}\left\|\left(I-T_{N}\right) U_{N-1} x-\left(I-T_{N}\right) U_{N-1} y\right\|^{2}\right) \\
& +\alpha_{N}\left(\kappa_{N}-\beta_{N}\right)\left\|\left(I-T_{N}\right) T_{N} U_{N-1} x-\left(I-T_{N}\right) T_{N} U_{N-1} y\right\|^{2} \\
& +\gamma_{N}\left\|U_{N-1} x-U_{N-1} y\right\|^{2}+\delta_{N}\|x-y\|^{2} \\
& -\beta_{N} \gamma_{N}\left\|\left(I-T_{N}\right) U_{N-1} x-\left(I-T_{N}\right) U_{N-1} y\right\|^{2} \\
& =\left(1-\delta_{N}\right)\left\|U_{N-1} x-U_{N-1} y\right\|^{2}+\left(1-\left(1-\delta_{N}\right)\right)\|x-y\|^{2} \\
& +\alpha_{N}\left(\kappa_{N}-\beta_{N}\right)\left\|\left(I-T_{N}\right) T_{N} U_{N-1} x-\left(I-T_{N}\right) T_{N} U_{N-1} y\right\|^{2} \\
& +\left(\left(\alpha_{N}+\beta_{N}\right) \kappa_{n}-\beta_{N} \gamma_{N}\right)\left\|\left(I-T_{N}\right) U_{N-1} x-\left(I-T_{N}\right) U_{N-1} y\right\|^{2} \\
& \leq\left(1-\delta_{N}\right)\left\|U_{N-1} x-U_{N-1} y\right\|^{2}+\left(1-\left(1-\delta_{N}\right)\right)\|x-y\|^{2} \\
& \vdots \\
& \leq\left(1-\delta_{N}\right)\left[\left(1-\delta_{N-1}\right)\left\|U_{N-2} x-U_{N-2} y\right\|^{2}+\left(1-\left(1-\delta_{N-1}\right)\right)\|x-y\|^{2}\right] \\
& +\left(1-\left(1-\delta_{N}\right)\right)\|x-y\|^{2} \\
& =\left(1-\delta_{N}\right)\left(1-\delta_{N-1}\right)\left\|U_{N-2} x-U_{N-2} y\right\|^{2}+\left(1-\left(1-\delta_{N}\right)\left(1-\delta_{N-1}\right)\right)\|x-y\|^{2}
\end{aligned}
$$




$$
\begin{aligned}
& \leq \prod_{i=2}^{N}\left(1-\delta_{i}\right)\left\|U_{1} x-U_{1} y\right\|^{2}+\left(1-\prod_{i=2}^{N}\left(1-\delta_{i}\right)\right)\|x-y\|^{2} \\
& =\prod_{i=2}^{N}\left(1-\delta_{i}\right)\left\|\alpha_{1}\left(T_{1}^{2} x-T_{1}^{2} y\right)+\beta_{1}\left(T_{1} x-T_{1} y\right)+\left(1-\alpha_{1}-\beta_{1}\right)(x-y)\right\|^{2} \\
& +\left(1-\prod_{i=2}^{N}\left(1-\delta_{i}\right)\right)\|x-y\|^{2} \\
& \leq \prod_{i=2}^{N}\left(1-\delta_{i}\right)\left[\alpha_{1}\left\|T_{1}^{2} x-T_{1}^{2} y\right\|^{2}+\beta_{1}\left\|T_{1} x-T_{1} y\right\|^{2}+\left(1-\alpha_{1}-\beta_{1}\right)\|x-y\|^{2}\right. \\
& \left.-\alpha_{1} \beta_{1}\left\|\left(I-T_{1}\right) T_{1} x-\left(I-T_{1}\right) T_{1} y\right\|^{2}-\beta_{1}\left(1-\alpha_{1}-\beta_{1}\right)\left\|\left(I-T_{1}\right) x-\left(I-T_{1}\right) y\right\|^{2}\right] \\
& +\left(1-\prod_{i=2}^{N}\left(1-\delta_{i}\right)\right)\|x-y\|^{2} \\
& \leq \prod_{i=2}^{N}\left(1-\delta_{i}\right)\left[\alpha_{1}\left(\left\|T_{1} x-T_{1} y\right\|^{2}+\kappa_{1}\left\|\left(I-T_{1}\right) T_{1} x-\left(I-T_{1}\right) T_{1} y\right\|^{2}\right)+\beta_{1}\left\|T_{1} x-T_{1} y\right\|^{2}\right. \\
& +\left(1-\alpha_{1}-\beta_{1}\right)\|x-y\|^{2}-\alpha_{1} \beta_{1}\left\|\left(I-T_{1}\right) T_{1} x-\left(I-T_{1}\right) T_{1} y\right\|^{2} \\
& \left.-\beta_{1}\left(1-\alpha_{1}-\beta_{1}\right)\left\|\left(I-T_{1}\right) x-\left(I-T_{1}\right) y\right\|^{2}\right] \\
& +\left(1-\prod_{i=2}^{N}\left(1-\delta_{i}\right)\right)\|x-y\|^{2} \\
& =\prod_{i=2}^{N}\left(1-\delta_{i}\right)\left[\left(\alpha_{1}+\beta_{1}\right)\left\|T_{1} x-T_{1} y\right\|^{2}+\alpha_{1}\left(\kappa_{1}-\beta_{1}\right)\left\|\left(I-T_{1}\right) T_{1} x-\left(I-T_{1}\right) T_{1} y\right\|^{2}\right. \\
& \left.+\left(1-\alpha_{1}-\beta_{1}\right)\|x-y\|^{2}-\beta_{1}\left(1-\alpha_{1}-\beta_{1}\right)\left\|\left(I-T_{1}\right) x-\left(I-T_{1}\right) y\right\|^{2}\right] \\
& +\left(1-\prod_{i=2}^{N}\left(1-\delta_{i}\right)\right)\|x-y\|^{2} \\
& \leq \prod_{i=2}^{N}\left(1-\delta_{i}\right)\left[\left(\alpha_{1}+\beta_{1}\right)\left(\|x-y\|^{2}+\kappa_{1}\left\|\left(I-T_{1}\right) x-\left(I-T_{1}\right) y\right\|^{2}\right)\right. \\
& +\alpha_{1}\left(\kappa_{1}-\beta_{1}\right)\left\|\left(I-T_{1}\right) T_{1} x-\left(I-T_{1}\right) T_{1} y\right\|^{2}+\left(1-\alpha_{1}-\beta_{1}\right)\|x-y\|^{2} \\
& \left.-\beta_{1}\left(1-\alpha_{1}-\beta_{1}\right)\left\|\left(I-T_{1}\right) x-\left(I-T_{1}\right) y\right\|^{2}\right]+\left(1-\prod_{i=2}^{N}\left(1-\delta_{i}\right)\right)\|x-y\|^{2} \\
& =\prod_{i=2}^{N}\left(1-\delta_{i}\right)\left[\|x-y\|^{2}+\alpha_{1}\left(\kappa_{1}-\beta_{1}\right)\left\|\left(I-T_{1}\right) T_{1} x-\left(I-T_{1}\right) T_{1} y\right\|^{2}\right. \\
& \left.+\left(\left(\alpha_{1}+\beta_{1}\right) \kappa_{1}-\beta_{1}\left(1-\alpha_{1}-\beta_{1}\right)\right)\left\|\left(I-T_{1}\right) x-\left(I-T_{1}\right) y\right\|^{2}\right] \\
& +\left(1-\prod_{i=2}^{N}\left(1-\delta_{i}\right)\right)\|x-y\|^{2}
\end{aligned}
$$




$$
\begin{aligned}
& \leq \prod_{i=2}^{N}\left(1-\delta_{i}\right)\|x-y\|^{2}+\left(1-\prod_{i=2}^{N}\left(1-\delta_{i}\right)\right)\|x-y\|^{2} \\
& =\|x-y\|^{2} .
\end{aligned}
$$

This completes the proof.

Remark 2.1 From the above proof, we can see that the mapping $G$ is quasi-nonexpansive under the conditions in Lemma 2.9, that is,

$$
\left\|G x-x^{*}\right\| \leq\left\|x-x^{*}\right\|, \quad \forall x \in C, x^{*} \in F(G) .
$$

Example 2.1 Let $T_{1}, T_{2}: \mathbb{R} \rightarrow \mathbb{R}$ be defined by

$$
T_{1} x= \begin{cases}x, & x \in(-\infty, 0] \\ -\frac{3}{2} x, & x \in[0,+\infty)\end{cases}
$$

and

$$
T_{2} x= \begin{cases}-2 x, & x \in(-\infty, 0] \\ x, & x \in[0,+\infty)\end{cases}
$$

Then we observe that $F\left(T_{1}\right)=(-\infty, 0]$ and $F\left(T_{2}\right)=[0,+\infty)$. Hence, $F\left(T_{1}\right) \cap F\left(T_{2}\right)=\{0\}$.

Firstly, we show that $T_{1}$ is a $\frac{1}{5}$-strictly pseudo-contractive mapping.

(1) If $x, y \in(-\infty, 0]$, then we have

$$
\left\|T_{1} x-T_{1} y\right\|^{2}=(x-y)^{2}
$$

and

$$
\left\|\left(I-T_{1}\right) x-\left(I-T_{1}\right) y\right\|^{2}=0 .
$$

From the above, then there exists $\kappa_{1} \in[0,1)$ such that

$$
\left\|T_{1} x-T_{1} y\right\|^{2} \leq\|x-y\|^{2}+\kappa_{1}\left\|\left(I-T_{1}\right) x-\left(I-T_{1}\right) y\right\|^{2} .
$$

(2) If $x, y \in[0,+\infty)$, then we have

$$
\left\|T_{1} x-T_{1} y\right\|^{2}=\left(-\frac{3}{2} x+\frac{3}{2} y\right)^{2}=\frac{9}{4}(x-y)^{2}
$$

and

$$
\left\|\left(I-T_{1}\right) x-\left(I-T_{1}\right) y\right\|^{2}=\left(\left(x+\frac{3}{2} x\right)-\left(y+\frac{3}{2} y\right)\right)^{2}=\frac{25}{4}(x-y)^{2} .
$$

From the above, then there exists $\kappa_{1} \in\left[\frac{1}{5}, 1\right)$ such that

$$
\left\|T_{1} x-T_{1} y\right\|^{2} \leq\|x-y\|^{2}+\kappa_{1}\left\|\left(I-T_{1}\right) x-\left(I-T_{1}\right) y\right\|^{2} .
$$


(3) If $x \in(-\infty, 0]$ and $y \in[0,+\infty)$, then we have

$$
\left\|T_{1} x-T_{1} y\right\|^{2}=\left(x+\frac{3}{2} y\right)^{2}
$$

and

$$
\left\|\left(I-T_{1}\right) x-\left(I-T_{1}\right) y\right\|^{2}=\left((x-x)-\left(y+\frac{3}{2} y\right)\right)^{2}=\frac{25}{4} y^{2} .
$$

Note that

$$
\left(x+\frac{3}{2} y\right)^{2}-(x-y)^{2}-\kappa_{1} \frac{25}{4} y^{2}=\left(\frac{5}{4}-\frac{25}{4} \kappa_{1}\right) y^{2}+5 x y .
$$

From the above, then there exists $\kappa_{1} \in\left[\frac{1}{5}, 1\right)$ such that

$$
\left\|T_{1} x-T_{1} y\right\|^{2} \leq\|x-y\|^{2}+\kappa_{1}\left\|\left(I-T_{1}\right) x-\left(I-T_{1}\right) y\right\|^{2} .
$$

Next, we show that $T_{2}$ is a $\frac{1}{3}$-strictly pseudo-contractive mapping.

(1) If $x, y \in(-\infty, 0]$, then we have

$$
\left\|T_{2} x-T_{2} y\right\|^{2}=(-2 x+2 y)^{2}=4(x-y)^{2}
$$

and

$$
\left\|\left(I-T_{2}\right) x-\left(I-T_{2}\right) y\right\|^{2}=((x+2 x)-(y+2 y))^{2}=9(x-y)^{2} .
$$

From the above, then there exists $\kappa_{2} \in\left[\frac{1}{3}, 1\right)$ such that

$$
\left\|T_{2} x-T_{2} y\right\|^{2} \leq\|x-y\|^{2}+\kappa_{2}\left\|\left(I-T_{2}\right) x-\left(I-T_{2}\right) y\right\|^{2} .
$$

(2) If $x, y \in[0,+\infty)$, then we have

$$
\left\|T_{2} x-T_{2} y\right\|^{2}=(x-y)^{2}
$$

and

$$
\left\|\left(I-T_{2}\right) x-\left(I-T_{2}\right) y\right\|^{2}=0 .
$$

From the above, then there exists $\kappa_{2} \in[0,1)$ such that

$$
\left\|T_{2} x-T_{2} y\right\|^{2} \leq\|x-y\|^{2}+\kappa_{2}\left\|\left(I-T_{2}\right) x-\left(I-T_{2}\right) y\right\|^{2} .
$$

(3) If $x \in(-\infty, 0]$ and $y \in[0,+\infty)$, then we have

$$
\left\|T_{2} x-T_{2} y\right\|^{2}=(-2 x-y)^{2}
$$


and

$$
\left\|\left(I-T_{2}\right) x-\left(I-T_{2}\right) y\right\|^{2}=((x+2 x)-(y-y))^{2}=9 x^{2} .
$$

Note that

$$
(-2 x-y)^{2}-(x-y)^{2}-9 \kappa_{2} x^{2}=\left(3-9 \kappa_{2}\right) x^{2}+6 x y .
$$

From the above, then there exists $\kappa_{2} \in\left[\frac{1}{3}, 1\right)$ such that

$$
\left\|T_{2} x-T_{2} y\right\|^{2} \leq\|x-y\|^{2}+\kappa_{2}\left\|\left(I-T_{2}\right) x-\left(I-T_{2}\right) y\right\|^{2} .
$$

Let

$$
\pi_{1}=\left(\frac{1}{5}, \frac{1}{5}, \frac{2}{5}, \frac{1}{5}\right),
$$

which satisfies condition (i) in Lemma 2.9. And

$$
T_{1}^{2} x= \begin{cases}x, & x \in(-\infty, 0] \\ -\frac{3}{2} x, & x \in[0,+\infty)\end{cases}
$$

Then

$$
U_{1} x=\frac{1}{5} T_{1}^{2} x+\frac{1}{5} T_{1} x+\frac{2}{5} x+\frac{1}{5} x= \begin{cases}x, & x \in(-\infty, 0], \\ 0, & x \in[0,+\infty) .\end{cases}
$$

Let

$$
\pi_{2}=\left(\frac{1}{7}, \frac{1}{3}, \frac{1}{2}, \frac{1}{42}\right)
$$

which satisfies condition (ii) in Lemma 2.9. Again, we have

$$
T_{2} U_{1} x= \begin{cases}-2 x, & x \in(-\infty, 0], \\ 0, & x \in[0,+\infty)\end{cases}
$$

and

$$
T_{2}^{2} U_{1} x= \begin{cases}-2 x, & x \in(-\infty, 0] \\ 0, & x \in[0,+\infty)\end{cases}
$$

Then

$$
\begin{aligned}
G x & =U_{2} x=\frac{1}{7} T_{2}^{2} U_{1} x+\frac{1}{3} T_{2} U_{1} x+\frac{1}{2} U_{1} x+\frac{1}{42} x \\
& = \begin{cases}-\frac{3}{7} x, & x \in(-\infty, 0], \\
\frac{1}{42} x, & x \in[0,+\infty) .\end{cases}
\end{aligned}
$$

From the above, we can get $F(G)=\{0\}$, that is, $F(G)=F\left(T_{1}\right) \cap F\left(T_{2}\right)$. 
Finally, we show that $G$ is nonexpansive.

(1) If $x, y \in(-\infty, 0]$, it is easy to see that

$$
\left|-\frac{3}{7} x+\frac{3}{7} y\right| \leq|x-y| .
$$

(2) If $x, y \in[0,+\infty)$, we have

$$
\left|\frac{1}{42} x-\frac{1}{42} y\right| \leq|x-y|
$$

(3) If $x \in(-\infty, 0]$ and $y \in[0,+\infty)$, then

$$
\begin{aligned}
\left|-\frac{3}{7} x-\frac{1}{42} y\right|^{2}-|x-y|^{2} \\
\quad=-\frac{40}{49} x^{2}-\frac{1,763}{1,764} y^{2}+\frac{99}{49} x y \\
\leq 0 \quad\left(\text { since } x \leq 0 \text { and } y \geq 0, \text { then } \frac{99}{49} x y \leq 0\right) .
\end{aligned}
$$

Hence,

$$
\left|-\frac{3}{7} x-\frac{1}{42} y\right| \leq|x-y|
$$

\section{Main results}

Theorem 3.1 Let $C$ be a nonempty closed convex subset of the real Hilbert space $H$. For every $i=1,2, \ldots, N$, let $T_{i}: C \rightarrow C$ be $\kappa_{i}$-strict pseudo-contractive mappings and $T: C \rightarrow C$ be a $\rho$-strictly pseudononspreading mapping for some $\rho \in[0,1)$. For $i=1,2, \ldots, N$, let $\pi_{i}=$ $\left(\alpha_{i}, \beta_{i}, \gamma_{i}, \delta_{i}\right)$, where $\alpha_{i}, \beta_{i}, \gamma_{i}, \delta_{i} \in[0,1], \alpha_{i}+\beta_{i}+\gamma_{i}+\delta_{i}=1$ and satisfy

(i) $\kappa_{1} \leq \beta_{1}<1-\kappa_{1}$ and $\alpha_{1}\left(\kappa_{1}+\beta_{1}\right)<\beta_{1}\left(1-\beta_{1}-\kappa_{1}\right)$;

(ii) $\beta_{i} \geq \kappa_{i}, \kappa_{i}<\gamma_{i}<1$ and $\kappa_{i} \alpha_{i} \leq \beta_{i} \gamma_{i}-\beta_{i} \kappa_{i}$ for $i=2,3, \ldots, N$.

Let $G$ be the G-mapping generated by $T_{1}, T_{2}, \ldots, T_{N}$ and $\pi_{1}, \pi_{2}, \ldots, \pi_{N}$. Assume that $\mathfrak{F}=$ $F(T) \cap \bigcap_{i=1}^{N} F\left(T_{i}\right) \neq \emptyset$. Pick any $u, x_{0} \in C$, let $\left\{x_{n}\right\}$ be a sequence generated by

$$
\left\{\begin{array}{l}
y_{n}=\left(1-s_{n}\right) x_{n}+s_{n} P_{C}\left(I-\lambda_{n}(I-T)\right) x_{n} \\
z_{n}=\left(1-t_{n}\right) x_{n}+t_{n} P_{C}\left(I-\lambda_{n}(I-T)\right) y_{n} \\
x_{n+1}=a_{n} u+b_{n} z_{n}+c_{n} G z_{n}
\end{array}\right.
$$

where $\left\{s_{n}\right\},\left\{t_{n}\right\},\left\{a_{n}\right\},\left\{b_{n}\right\},\left\{c_{n}\right\} \subset[0,1]$ and $\left\{\lambda_{n}\right\} \subset(0,1-\rho)$ satisfy the following conditions:

(1) $a_{n}+b_{n}+c_{n}=1$;

(2) $\lim _{n \rightarrow \infty} a_{n}=0$ and $\sum_{n=0}^{\infty} a_{n}=\infty$;

(3) $\liminf _{n \rightarrow \infty} b_{n}>0$ and $\liminf _{n \rightarrow \infty} c_{n}>0$;

(4) $\sum_{n=0}^{\infty} \lambda_{n}<\infty$;

(5) $\sum_{n=0}^{\infty}\left|\lambda_{n+1}-\lambda_{n}\right|, \sum_{n=0}^{\infty}\left|s_{n+1}-s_{n}\right|, \sum_{n=0}^{\infty}\left|t_{n+1}-t_{n}\right|, \sum_{n=0}^{\infty}\left|a_{n+1}-a_{n}\right|$, $\sum_{n=0}^{\infty}\left|b_{n+1}-b_{n}\right|, \sum_{n=0}^{\infty}\left|c_{n+1}-c_{n}\right|<\infty$.

Then $\left\{x_{n}\right\}$ converges strongly to $\bar{x}=P_{\mathfrak{F}} u$. 
Proof Step 1. Firstly, we show that $L$ is bounded, where

$$
\begin{aligned}
L= & \max _{n \in \mathbb{N}}\left\{\|u\|,\left\|x_{n}\right\|,\left\|z_{n}\right\|,\left\|G z_{n}\right\|,\left\|P_{C}\left(I-\lambda_{n}(I-T)\right) x_{n}\right\|,\left\|P_{C}\left(I-\lambda_{n}(I-T)\right) y_{n}\right\|,\right. \\
& \left\|(I-T) x_{n}-(I-T) x_{n-1}\right\|,\left\|(I-T) y_{n}-(I-T) y_{n-1}\right\|, \\
& \left.\left\|(I-T) x_{n}\right\|,\left\|(I-T) y_{n}\right\|\right\} .
\end{aligned}
$$

Indeed, take $p \in \mathfrak{F}$ arbitrarily. From (3.1), we have

$$
\begin{aligned}
\left\|x_{n+1}-p\right\| & =\left\|a_{n} u+b_{n} z_{n}+c_{n} G z_{n}-p\right\| \\
& =\left\|a_{n}(u-p)+b_{n}\left(z_{n}-p\right)+c_{n}\left(G z_{n}-p\right)\right\| \\
& \leq a_{n}\|u-p\|+b_{n}\left\|z_{n}-p\right\|+c_{n}\left\|G z_{n}-p\right\| \\
& \leq a_{n}\|u-p\|+b_{n}\left\|z_{n}-p\right\|+c_{n}\left\|z_{n}-p\right\| \\
& =a_{n}\|u-p\|+\left(1-a_{n}\right)\left\|z_{n}-p\right\| .
\end{aligned}
$$

From Lemma 2.3 and (3.1), we have

$$
\begin{aligned}
\left\|z_{n}-p\right\| & =\left\|\left(1-t_{n}\right) x_{n}+t_{n} P_{C}\left(I-\lambda_{n}(I-T)\right) y_{n}-p\right\| \\
& \leq\left(1-t_{n}\right)\left\|x_{n}-p\right\|+t_{n}\left\|P_{C}\left(I-\lambda_{n}(I-T)\right) y_{n}-p\right\| \\
& \leq\left(1-t_{n}\right)\left\|x_{n}-p\right\|+t_{n}\left\|y_{n}-p\right\|,
\end{aligned}
$$

and

$$
\begin{aligned}
\left\|y_{n}-p\right\| & =\left\|\left(1-s_{n}\right) x_{n}+s_{n} P_{C}\left(I-\lambda_{n}(I-T)\right) x_{n}-p\right\| \\
& \leq\left(1-s_{n}\right)\left\|x_{n}-p\right\|+s_{n}\left\|P_{C}\left(I-\lambda_{n}(I-T)\right) x_{n}-p\right\| \\
& \leq\left(1-s_{n}\right)\left\|x_{n}-p\right\|+s_{n}\left\|x_{n}-p\right\| \\
& =\left\|x_{n}-p\right\| .
\end{aligned}
$$

Substituting (3.4) and (3.5) into (3.3), we obtain that

$$
\left\|x_{n+1}-p\right\| \leq a_{n}\|u-p\|+\left(1-a_{n}\right)\left\|x_{n}-p\right\| .
$$

From (3.6), we can see by induction that

$$
\left\|x_{n+1}-p\right\| \leq \max \left\{\|u-p\|,\left\|x_{0}-p\right\|\right\}, \quad \forall n \geq 0
$$

This implies that $\left\{x_{n}\right\}$ is bounded. Then $\left\{y_{n}\right\},\left\{z_{n}\right\}$ and $\left\{G z_{n}\right\}$ are bounded. From Lemma 2.3 and the boundedness of $\left\{x_{n}\right\}$ and $\left\{y_{n}\right\}$, it can be seen that $\left\{P_{C}\left(I-\lambda_{n}(I-T)\right) x_{n}\right\}$ and $\left\{P_{C}(I-\right.$ $\left.\left.\lambda_{n}(I-T)\right) y_{n}\right\}$ are bounded. And from Lemma 2.4, we also have that $\left\{(I-T) x_{n}-(I-T) x_{n-1}\right\}$ and $\left\{(I-T) y_{n}-(I-T) y_{n-1}\right\}$ are bounded. Hence, $L$ is bounded.

Step 2. Next, we prove that $\lim _{n \rightarrow \infty}\left\|x_{n+1}-x_{n}\right\|=0$. 
From (3.1), it follows that

$$
\begin{aligned}
& \left\|x_{n+1}-x_{n}\right\| \\
& =\left\|a_{n} u+b_{n} z_{n}+c_{n} G z_{n}-\left(a_{n-1} u+b_{n-1} z_{n-1}+c_{n-1} G z_{n-1}\right)\right\| \\
& =\|\left(a_{n}-a_{n-1}\right) u+b_{n}\left(z_{n}-z_{n-1}\right)+\left(b_{n}-b_{n-1}\right) z_{n-1}+c_{n}\left(G z_{n}-G z_{n-1}\right) \\
& +\left(c_{n}-c_{n-1}\right) G z_{n-1} \| \\
& \leq\left|a_{n}-a_{n-1}\right|\|u\|+b_{n}\left\|z_{n}-z_{n-1}\right\|+\left|b_{n}-b_{n-1}\right|\left\|z_{n-1}\right\|+c_{n}\left\|G z_{n}-G z_{n-1}\right\| \\
& +\left|c_{n}-c_{n-1}\right||| G z_{n-1}|| \\
& \leq\left|a_{n}-a_{n-1}\right| L+b_{n}\left\|z_{n}-z_{n-1}||+\left|b_{n}-b_{n-1}\right| L+c_{n}\right\| z_{n}-z_{n-1} \|+\left|c_{n}-c_{n-1}\right| L \\
& =\left(1-a_{n}\right)\left\|z_{n}-z_{n-1}\right\|+\left|a_{n}-a_{n-1}\right| L+\left|b_{n}-b_{n-1}\right| L+\left|c_{n}-c_{n-1}\right| L, \\
& =\|\left(1-t_{n}\right) x_{n}+t_{n} P_{C}\left(I-\lambda_{n}(I-T)\right) y_{n}-\left(\left(1-t_{n-1}\right) x_{n-1}\right. \\
& \left.+t_{n-1} P_{C}\left(I-\lambda_{n-1}(I-T)\right) y_{n-1}\right) \| \\
& \leq\left\|\left(1-t_{n}\right) x_{n}-\left(1-t_{n-1}\right) x_{n-1}\right\|+\| t_{n} P_{C}\left(I-\lambda_{n}(I-T)\right) y_{n} \\
& -t_{n-1} P_{C}\left(I-\lambda_{n-1}(I-T)\right) y_{n-1} \| \\
& \leq\left(1-t_{n}\right)\left\|x_{n}-x_{n-1}\right\|+\left|t_{n}-t_{n-1}\right|\left\|x_{n-1}\right\|+t_{n} \| P_{C}\left(I-\lambda_{n}(I-T)\right) y_{n} \\
& -P_{C}\left(I-\lambda_{n-1}(I-T)\right) y_{n-1} \| \\
& +\left|t_{n}-t_{n-1}\right|\left\|P_{C}\left(I-\lambda_{n-1}(I-T)\right) y_{n-1}\right\| \\
& \leq\left(1-t_{n}\right)\left\|x_{n}-x_{n-1}\right\|+\left|t_{n}-t_{n-1}\right| L+t_{n}\left\|\left(I-\lambda_{n}(I-T)\right) y_{n}-\left(I-\lambda_{n-1}(I-T)\right) y_{n-1}\right\| \\
& +\left|t_{n}-t_{n-1}\right| L \\
& \leq\left(1-t_{n}\right)\left\|x_{n}-x_{n-1}\right\|+2\left|t_{n}-t_{n-1}\right| L+t_{n}\left\|y_{n}-y_{n-1}\right\| \\
& +t_{n}\left\|\lambda_{n}(I-T) y_{n}-\lambda_{n}(I-T) y_{n-1}+\lambda_{n}(I-T) y_{n-1}-\lambda_{n-1}(I-T) y_{n-1}\right\| \\
& \leq\left(1-t_{n}\right)\left\|x_{n}-x_{n-1}\right\|+2\left|t_{n}-t_{n-1}\right| L+t_{n}\left\|y_{n}-y_{n-1}\right\| \\
& +t_{n} \lambda_{n}\left\|(I-T) y_{n}-(I-T) y_{n-1}\right\|+t_{n}\left|\lambda_{n}-\lambda_{n-1}\right|\left\|(I-T) y_{n-1}\right\| \\
& \leq\left(1-t_{n}\right)\left\|x_{n}-x_{n-1}\right\|+t_{n}\left\|y_{n}-y_{n-1}\right\|+2\left|t_{n}-t_{n-1}\right| L+t_{n} \lambda_{n} L+t_{n}\left|\lambda_{n}-\lambda_{n-1}\right| L,
\end{aligned}
$$

and

$$
\begin{aligned}
& \left\|y_{n+1}-y_{n}\right\| \\
& =\|\left(1-s_{n}\right) x_{n}+s_{n} P_{C}\left(I-\lambda_{n}(I-T)\right) x_{n}-\left(\left(1-s_{n-1}\right) x_{n-1}\right. \\
& \left.\quad+s_{n-1} P_{C}\left(I-\lambda_{n-1}(I-T)\right) x_{n-1}\right) \| \\
& \leq\left\|\left(1-s_{n}\right) x_{n}-\left(1-s_{n-1}\right) x_{n-1}\right\|+\| s_{n} P_{C}\left(I-\lambda_{n}(I-T)\right) x_{n} \\
& \quad-s_{n-1} P_{C}\left(I-\lambda_{n-1}(I-T)\right) x_{n-1} \| \\
& \leq \\
& \leq
\end{aligned}
$$




$$
\begin{aligned}
& -P_{C}\left(I-\lambda_{n-1}(I-T)\right) x_{n-1} \| \\
& +\left|s_{n}-s_{n-1}\right|\left\|P_{C}\left(I-\lambda_{n-1}(I-T)\right) x_{n-1}\right\| \\
\leq & \left(1-s_{n}\right)\left\|x_{n}-x_{n-1}\right\|+\left|s_{n}-s_{n-1}\right| L+s_{n}\left\|\left(I-\lambda_{n}(I-T)\right) x_{n}-\left(I-\lambda_{n-1}(I-T)\right) x_{n-1}\right\| \\
& +\left|s_{n}-s_{n-1}\right| L \\
\leq & \left(1-s_{n}\right)\left\|x_{n}-x_{n-1}\right\|+2\left|s_{n}-s_{n-1}\right| L+s_{n}\left\|x_{n}-x_{n-1}\right\| \\
& +s_{n}\left\|\lambda_{n}(I-T) x_{n}-\lambda_{n}(I-T) x_{n-1}+\lambda_{n}(I-T) x_{n-1}-\lambda_{n-1}(I-T) x_{n-1}\right\| \\
\leq & \left\|x_{n}-x_{n-1}\right\|+2\left|s_{n}-s_{n-1}\right| L+s_{n} \lambda_{n}\left\|(I-T) x_{n}-(I-T) x_{n-1}\right\| \\
& +s_{n}\left|\lambda_{n}-\lambda_{n-1}\right|\left\|(I-T) x_{n-1}\right\| \\
\leq & \left\|x_{n}-x_{n-1}\right\|+2\left|s_{n}-s_{n-1}\right| L+s_{n} \lambda_{n} L+s_{n}\left|\lambda_{n}-\lambda_{n-1}\right| L
\end{aligned}
$$

Substituting (3.9) and (3.10) into (3.8), we can get that

$$
\begin{aligned}
\| x_{n+1} & -x_{n} \| \\
\leq & \left(1-a_{n}\right)\left\|z_{n}-z_{n-1}\right\|+\left|a_{n}-a_{n-1}\right| L+\left|b_{n}-b_{n-1}\right| L+\left|c_{n}-c_{n-1}\right| L \\
\leq & \left(1-a_{n}\right)\left[\left(1-t_{n}\right)\left\|x_{n}-x_{n-1}\right\|+t_{n}\left\|y_{n}-y_{n-1}\right\|+2\left|t_{n}-t_{n-1}\right| L\right. \\
& \left.+t_{n} \lambda_{n} L+t_{n}\left|\lambda_{n}-\lambda_{n-1}\right| L\right]+\left|a_{n}-a_{n-1}\right| L+\left|b_{n}-b_{n-1}\right| L+\left|c_{n}-c_{n-1}\right| L \\
\leq & \left(1-a_{n}\right)\left[\left(1-t_{n}\right)\left\|x_{n}-x_{n-1}\right\|+t_{n}\left(\left\|x_{n}-x_{n-1}\right\|+2\left|s_{n}-s_{n-1}\right| L\right.\right. \\
& \left.\left.+s_{n} \lambda_{n} L+s_{n}\left|\lambda_{n}-\lambda_{n-1}\right| L\right)\right] \\
& +2\left(1-a_{n}\right)\left|t_{n}-t_{n-1}\right| L+\left(1-a_{n}\right) t_{n} \lambda_{n} L+\left(1-a_{n}\right) t_{n}\left|\lambda_{n}-\lambda_{n-1}\right| L \\
& +\left|a_{n}-a_{n-1}\right| L+\left|b_{n}-b_{n-1}\right| L+\left|c_{n}-c_{n-1}\right| L \\
= & \left(1-a_{n}\right)\left\|x_{n}-x_{n-1}\right\|+2\left(1-a_{n}\right) t_{n}\left|s_{n}-s_{n-1}\right| L+\left(1-a_{n}\right) t_{n} s_{n} \lambda_{n} L \\
& +\left(1-a_{n}\right) t_{n} s_{n}\left|\lambda_{n}-\lambda_{n-1}\right| L \\
& +2\left(1-a_{n}\right)\left|t_{n}-t_{n-1}\right| L+\left(1-a_{n}\right) t_{n} \lambda_{n} L+\left(1-a_{n}\right) t_{n}\left|\lambda_{n}-\lambda_{n-1}\right| L \\
& +\left|a_{n}-a_{n-1}\right| L+\left|b_{n}-b_{n-1}\right| L+\left|c_{n}-c_{n-1}\right| L \\
= & \left(1-a_{n}\right)\left\|x_{n}-x_{n-1}\right\|+\theta_{n},
\end{aligned}
$$

where

$$
\begin{aligned}
\theta_{n}= & 2\left(1-a_{n}\right) t_{n}\left|s_{n}-s_{n-1}\right| L+\left(1-a_{n}\right) t_{n} s_{n} \lambda_{n} L+\left(1-a_{n}\right) t_{n} s_{n}\left|\lambda_{n}-\lambda_{n-1}\right| L \\
& +2\left(1-a_{n}\right)\left|t_{n}-t_{n-1}\right| L+\left(1-a_{n}\right) t_{n} \lambda_{n} L+\left(1-a_{n}\right) t_{n}\left|\lambda_{n}-\lambda_{n-1}\right| L \\
& +\left|a_{n}-a_{n-1}\right| L+\left|b_{n}-b_{n-1}\right| L+\left|c_{n}-c_{n-1}\right| L .
\end{aligned}
$$

By the conditions in Theorem 3.1, we can get that

$$
\sum_{n=0}^{\infty} \theta_{n}<\infty
$$


Thus, from Lemma 2.5 and (3.11), we have

$$
\lim _{n \rightarrow \infty}\left\|x_{n+1}-x_{n}\right\|=0
$$

Step 3. In this step, we will show that $\lim _{n \rightarrow \infty}\left\|G z_{n}-z_{n}\right\|=0$ and $\lim _{n \rightarrow \infty}\left\|x_{n}-z_{n}\right\|=0$. From Lemma 2.1, (3.1), (3.4) and (3.5), we have

$$
\begin{aligned}
\left\|x_{n+1}-p\right\|^{2}= & \left\|a_{n} u+b_{n} z_{n}+c_{n} G z_{n}-p\right\|^{2} \\
= & \left\|a_{n}(u-p)+b_{n}\left(z_{n}-p\right)+c_{n}\left(G z_{n}-p\right)\right\|^{2} \\
= & a_{n}\|u-p\|^{2}+b_{n}\left\|z_{n}-p\right\|^{2}+c_{n}\left\|G z_{n}-p\right\|^{2} \\
& -a_{n} b_{n}\left\|u-z_{n}\right\|^{2}-a_{n} c_{n}\left\|u-G z_{n}\right\|^{2}-b_{n} c_{n}\left\|G z_{n}-z_{n}\right\|^{2} \\
\leq & a_{n}\|u-p\|^{2}+b_{n}\left\|z_{n}-p\right\|^{2}+c_{n}\left\|G z_{n}-p\right\|^{2}-b_{n} c_{n}\left\|G z_{n}-z_{n}\right\|^{2} \\
\leq & a_{n}\|u-p\|^{2}+b_{n}\left\|z_{n}-p\right\|^{2}+c_{n}\left\|z_{n}-p\right\|^{2}-b_{n} c_{n}\left\|G z_{n}-z_{n}\right\|^{2} \\
\leq & a_{n}\|u-p\|^{2}+\left(1-a_{n}\right)\left\|x_{n}-p\right\|^{2}-b_{n} c_{n}\left\|G z_{n}-z_{n}\right\|^{2} \\
\leq & a_{n}\|u-p\|^{2}+\left\|x_{n}-p\right\|^{2}-b_{n} c_{n}\left\|G z_{n}-z_{n}\right\|^{2},
\end{aligned}
$$

which implies that

$$
\begin{aligned}
b_{n} c_{n}\left\|G z_{n}-z_{n}\right\|^{2} & \leq a_{n}\|u-p\|^{2}+\left\|x_{n}-p\right\|^{2}-\left\|x_{n+1}-p\right\|^{2} \\
& \leq a_{n}\|u-p\|^{2}+\left(\left\|x_{n}-p\right\|+\left\|x_{n+1}-p\right\|\right)\left\|x_{n+1}-x_{n}\right\| .
\end{aligned}
$$

Since $\liminf _{n \rightarrow \infty} b_{n}>0, \liminf _{n \rightarrow \infty} c_{n}>0, \lim _{n \rightarrow \infty} a_{n}=0, \lim _{n \rightarrow \infty}\left\|x_{n+1}-x_{n}\right\|=0$ and by the boundedness of $\|u-p\|$ and $\left\{x_{n}\right\}$, we have

$$
\lim _{n \rightarrow \infty}\left\|G z_{n}-z_{n}\right\|=0
$$

Again,

$$
\begin{aligned}
\left\|x_{n}-z_{n}\right\| & \leq\left\|x_{n}-x_{n+1}\right\|+\left\|x_{n+1}-z_{n}\right\| \\
& \leq\left\|x_{n}-x_{n+1}\right\|+\left\|a_{n} u+b_{n} z_{n}+c_{n} G z_{n}-z_{n}\right\| \\
& \leq\left\|x_{n}-x_{n+1}\right\|+a_{n}\left\|u-z_{n}\right\|+c_{n}\left\|G z_{n}-z_{n}\right\| .
\end{aligned}
$$

Thus,

$$
\lim _{n \rightarrow \infty}\left\|x_{n}-z_{n}\right\|=0
$$

Step 4 . Now, we prove that $\limsup _{n \rightarrow \infty}\left\langle u-\bar{x}, x_{n}-\bar{x}\right\rangle \leq 0$, where $\bar{x}=P_{\mathfrak{F}} u$.

Take a subsequence $\left\{x_{n_{i}}\right\}$ of $\left\{x_{n}\right\}$ such that

$$
\limsup _{n \rightarrow \infty}\left\langle u-\bar{x}, x_{n}-\bar{x}\right\rangle=\lim _{n \rightarrow \infty}\left\langle u-\bar{x}, x_{n_{i}}-\bar{x}\right\rangle .
$$

Since $\left\{x_{n}\right\}$ is bounded, there exists a subsequence of $\left\{x_{n}\right\}$, which converges weakly to $x^{*}$. Without loss of generality, we may assume that $x_{n_{i}} \rightarrow x^{*}$. From (3.19), we have $z_{n_{i}} \rightarrow x^{*}$. 
From (3.17) and Lemma 2.7, we have $x^{*}=G x^{*}$, that is, $x^{*} \in F(G)$. Since $x_{n_{i}} \rightarrow x^{*}$, then $x^{*} \in F(T)$. In fact, if $x^{*} \notin F(T)$, then $T x^{*} \neq x^{*}$. Thus,

$$
\left(I-\lambda_{n_{i}}(I-T)\right) x^{*} \neq x^{*}
$$

By Lemma 2.8, we have

$$
\begin{aligned}
\liminf _{i \rightarrow \infty}\left\|x_{n_{i}}-x^{*}\right\| & <\liminf _{i \rightarrow \infty}\left\|x_{n_{i}}-\left(I-\lambda_{n_{i}}(I-T)\right) x^{*}\right\| \\
& \leq \liminf _{i \rightarrow \infty}\left(\left\|x_{n_{i}}-x^{*}\right\|+\lambda_{n_{i}}\left\|(I-T) x^{*}\right\|\right) \\
& \leq \liminf _{i \rightarrow \infty}\left\|x_{n_{i}}-x^{*}\right\| .
\end{aligned}
$$

This is a contradiction. Therefore,

$$
x^{*} \in \mathfrak{F}=F(T) \cap \bigcap_{i=1}^{N} F\left(T_{i}\right) .
$$

This together with the property of metric projection implies that

$$
\limsup _{n \rightarrow \infty}\left\langle u-\bar{x}, x_{n}-\bar{x}\right\rangle=\lim _{n \rightarrow \infty}\left\langle u-\bar{x}, x_{n_{i}}-\bar{x}\right\rangle=\left\langle u-\bar{x}, x^{*}-\bar{x}\right\rangle \leq 0 .
$$

Step 5 . Finally, we will show that $x_{n} \rightarrow \bar{x}$ as $n \rightarrow \infty$.

$$
\begin{aligned}
&\left\|x_{n+1}-\bar{x}\right\|^{2} \\
&=\left\langle a_{n} u+b_{n} z_{n}+c_{n} G z_{n}-\bar{x}, x_{n+1}-\bar{x}\right\rangle \\
&=a_{n}\left\langle u-\bar{x}, x_{n+1}-\bar{x}\right\rangle+b_{n}\left\langle z_{n}-\bar{x}, x_{n+1}-\bar{x}\right\rangle+c_{n}\left\langle G z_{n}-\bar{x}, x_{n+1}-\bar{x}\right\rangle \\
& \leq a_{n}\left\langle u-\bar{x}, x_{n+1}-\bar{x}\right\rangle+b_{n}\left\|z_{n}-\bar{x}\right\|\left\|x_{n+1}-\bar{x}\right\|+c_{n}\left\|G z_{n}-\bar{x}\right\|\left\|x_{n+1}-\bar{x}\right\| \\
& \leq a_{n}\left\langle u-\bar{x}, x_{n+1}-\bar{x}\right\rangle+b_{n}\left\|z_{n}-\bar{x}\right\|\left\|x_{n+1}-\bar{x}\right\|+c_{n}\left\|z_{n}-\bar{x}\right\|\left\|x_{n+1}-\bar{x}\right\| \\
& \leq a_{n}\left\langle u-\bar{x}, x_{n+1}-\bar{x}\right\rangle+b_{n}\left\|x_{n}-\bar{x}\right\|\left\|x_{n+1}-\bar{x}\right\|+c_{n}\left\|x_{n}-\bar{x}\right\|\left\|x_{n+1}-\bar{x}\right\| \\
& \leq a_{n}\left\langle u-\bar{x}, x_{n+1}-\bar{x}\right\rangle+\frac{b_{n}}{2}\left(\left\|x_{n}-\bar{x}\right\|^{2}+\left\|x_{n+1}-\bar{x}\right\|^{2}\right) \\
&+\frac{c_{n}}{2}\left(\left\|x_{n}-\bar{x}\right\|^{2}+\left\|x_{n+1}-\bar{x}\right\|^{2}\right),
\end{aligned}
$$

that is,

$$
\left\|x_{n+1}-\bar{x}\right\|^{2} \leq\left(1-\frac{2 a_{n}}{1+a_{n}}\right)\left\|x_{n}-\bar{x}\right\|^{2}+\frac{2 a_{n}}{1+a_{n}}\left\langle u-\bar{x}, x_{n+1}-\bar{x}\right\rangle .
$$

It is clear that $\sum_{n=0}^{\infty} \frac{2 a_{n}}{1+a_{n}}=\infty$. Hence, applying (3.24), (3.26) and Lemma 2.6, we obtain immediately that

$$
\lim _{n \rightarrow \infty}\left\|x_{n+1}-\bar{x}\right\|^{2}=0
$$

that is, $x_{n} \rightarrow \bar{x}$ as $n \rightarrow \infty$. This completes the proof. 


\section{Application}

From Theorem 3.1, we can obtain the following theorem.

Theorem 4.1 Let $C$ be a nonempty closed convex subset of the real Hilbert space H. For every $i=1,2, \ldots, N$, let $T_{i}: C \rightarrow C$ be nonexpansive mappings and $T: C \rightarrow C$ be a $\rho$-strictly pseudononspreading mapping for some $\rho \in[0,1)$. For $i=1,2, \ldots, N$, let $\pi_{i}=\left(\alpha_{i}, \beta_{i}, \gamma_{i}, \delta_{i}\right)$, where $\alpha_{i}, \beta_{i}, \gamma_{i}, \delta_{i} \in[0,1], \alpha_{i}+\beta_{i}+\gamma_{i}+\delta_{i}=1$ and satisfy

(i) $0<\beta_{1}<1$ and $\alpha_{1}<1-\beta_{1}$;

(ii) $0<\gamma_{i}<1$ for $i=2,3, \ldots, N$.

Let $G$ be the G-mapping generated by $T_{1}, T_{2}, \ldots, T_{N}$ and $\pi_{1}, \pi_{2}, \ldots, \pi_{N}$. Assume that $\mathfrak{F}=$ $F(T) \cap \bigcap_{i=1}^{N} F\left(T_{i}\right) \neq \emptyset$. Pick any $u, x_{0} \in C$, let $\left\{x_{n}\right\}$ be a sequence generated by

$$
\left\{\begin{array}{l}
y_{n}=\left(1-s_{n}\right) x_{n}+s_{n} P_{C}\left(I-\lambda_{n}(I-T)\right) x_{n}, \\
z_{n}=\left(1-t_{n}\right) x_{n}+t_{n} P_{C}\left(I-\lambda_{n}(I-T)\right) y_{n}, \\
x_{n+1}=a_{n} u+b_{n} z_{n}+c_{n} G z_{n}
\end{array}\right.
$$

where $\left\{s_{n}\right\},\left\{t_{n}\right\},\left\{a_{n}\right\},\left\{b_{n}\right\},\left\{c_{n}\right\} \subset[0,1]$ and $\left\{\lambda_{n}\right\} \subset(0,1-\rho)$ satisfy the following conditions:

(1) $a_{n}+b_{n}+c_{n}=1$

(2) $\lim _{n \rightarrow \infty} a_{n}=0$ and $\sum_{n=0}^{\infty} a_{n}=\infty$;

(3) $\liminf _{n \rightarrow \infty} b_{n}>0$ and $\liminf _{n \rightarrow \infty} c_{n}>0$;

(4) $\sum_{n=0}^{\infty} \lambda_{n}<\infty$;

(5) $\sum_{n=0}^{\infty}\left|\lambda_{n+1}-\lambda_{n}\right|, \sum_{n=0}^{\infty}\left|s_{n+1}-s_{n}\right|, \sum_{n=0}^{\infty}\left|t_{n+1}-t_{n}\right|, \sum_{n=0}^{\infty}\left|a_{n+1}-a_{n}\right|$, $\sum_{n=0}^{\infty}\left|b_{n+1}-b_{n}\right|, \sum_{n=0}^{\infty}\left|c_{n+1}-c_{n}\right|<\infty$.

Then $\left\{x_{n}\right\}$ converges strongly to $\bar{x}=P_{\mathfrak{F}} u$.

Lemma 4.1 [48] Let $C$ be a nonempty closed convex subset of $H$ and $T: C \rightarrow H$ be a $\xi$ inverse-strongly monotone mapping, then for all $x, y \in C$ and $\eta>0$, we have

$$
\begin{aligned}
\|(I-\eta T) x-(I-\eta T) y\|^{2} & =\|(x-y)-\eta(T x-T y)\|^{2} \\
& =\|x-y\|^{2}-2 \eta\langle T x-T y, x-y\rangle+\eta^{2}\|T x-T y\|^{2} \\
& \leq\|x-y\|^{2}+\eta(\eta-2 \xi)\|T x-T y\|^{2} .
\end{aligned}
$$

So, if $0<\eta \leq 2 \xi$, then $I-\eta T$ is a nonexpansive mapping from $C$ to $H$.

From Theorem 4.1, Lemmas 2.2 and 4.1, we have the following result.

Theorem 4.2 Let $C$ be a nonempty closed convex subset of the real Hilbert space $H$. For every $i=1,2, \ldots, N$, let $B_{i}: C \rightarrow H$ be $\xi_{i}$-inverse-strongly monotone mappings and $T: C \rightarrow$ $C$ be a $\rho$-strictly pseudononspreading mapping for some $\rho \in[0,1)$. For $i=1,2, \ldots, N$, let $T_{i}: C \rightarrow C$ be defined by $T_{i} x=P_{C}\left(I-\eta_{i} B_{i}\right) x$ for every $x \in C$ and $\eta_{i} \in\left(0,2 \xi_{i}\right)$, and let $\pi_{i}=$ $\left(\alpha_{i}, \beta_{i}, \gamma_{i}, \delta_{i}\right)$, where $\alpha_{i}, \beta_{i}, \gamma_{i}, \delta_{i} \in[0,1], \alpha_{i}+\beta_{i}+\gamma_{i}+\delta_{i}=1$ and satisfy

(i) $0<\beta_{1}<1$ and $\alpha_{1}<1-\beta_{1}$;

(ii) $0<\gamma_{i}<1$ for $i=2,3, \ldots, N$. 
Let $G$ be the G-mapping generated by $T_{1}, T_{2}, \ldots, T_{N}$ and $\pi_{1}, \pi_{2}, \ldots, \pi_{N}$. Assume that $\mathfrak{F}=$ $F(T) \cap \bigcap_{i=1}^{N} F\left(T_{i}\right) \neq \emptyset$. Pick any $u, x_{0} \in C$, let $\left\{x_{n}\right\}$ be a sequence generated by

$$
\left\{\begin{array}{l}
y_{n}=\left(1-s_{n}\right) x_{n}+s_{n} P_{C}\left(I-\lambda_{n}(I-T)\right) x_{n} \\
z_{n}=\left(1-t_{n}\right) x_{n}+t_{n} P_{C}\left(I-\lambda_{n}(I-T)\right) y_{n}, \\
x_{n+1}=a_{n} u+b_{n} z_{n}+c_{n} G z_{n}
\end{array}\right.
$$

where $\left\{s_{n}\right\},\left\{t_{n}\right\},\left\{a_{n}\right\},\left\{b_{n}\right\},\left\{c_{n}\right\} \subset[0,1]$ and $\left\{\lambda_{n}\right\} \subset(0,1-\rho)$ satisfy the following conditions:

(1) $a_{n}+b_{n}+c_{n}=1$;

(2) $\lim _{n \rightarrow \infty} a_{n}=0$ and $\sum_{n=0}^{\infty} a_{n}=\infty$;

(3) $\liminf _{n \rightarrow \infty} b_{n}>0$ and $\liminf _{n \rightarrow \infty} c_{n}>0$;

(4) $\sum_{n=0}^{\infty} \lambda_{n}<\infty$;

(5) $\sum_{n=0}^{\infty}\left|\lambda_{n+1}-\lambda_{n}\right|, \sum_{n=0}^{\infty}\left|s_{n+1}-s_{n}\right|, \sum_{n=0}^{\infty}\left|t_{n+1}-t_{n}\right|, \sum_{n=0}^{\infty}\left|a_{n+1}-a_{n}\right|$, $\sum_{n=0}^{\infty}\left|b_{n+1}-b_{n}\right|, \sum_{n=0}^{\infty}\left|c_{n+1}-c_{n}\right|<\infty$.

Then $\left\{x_{n}\right\}$ converges strongly to $\bar{x}=P_{\mathfrak{F}} u$.

\section{Competing interests}

The authors declare that they have no competing interests.

\section{Authors' contributions}

All authors contributed equally to the writing of this paper. All authors read and approved the final manuscript.

\section{Acknowledgements}

The authors are most grateful to the anonymous referees for their constructive comments and helpful suggestions, which greatly improved the original paper. The work is supported by the National Natural Science Foundation of China (11071041, 11201074), Fujian Natural Science Foundation (2013J01006, 2015J01578) and R\&D of Key Instruments and Technologies for Deep Resources Prospecting (the National R\&D Projects for Key Scientific Instruments) under grant number ZDYZ2012-1-02-04

Received: 9 February 2015 Accepted: 25 June 2015 Published online: 16 July 2015

\section{References}

1. Butnariu, D, Censor, Y, Gurfil, P, Hadar, E: On the behavior of subgradient projections methods for convex feasibility problem in Euclidean spaces. SIAM J. Optim. 19(2), 786-807 (2008)

2. Măruşter, S, Popirlan, C: On the Mann-type iteration and the convex feasibility problem. Comput. Appl. Math. 212(2), 390-396 (2008)

3. Masad, E, Reich, S: A note on the multiple-set split convex feasibility problem in Hilbert space. J. Nonlinear Convex Anal. 8, 367-371 (2007)

4. Xu, HK: A variable Krasnosel'skii-Mann algorithm and the multiple-set split feasibility problem. Inverse Probl. 22 2021-2034 (2006)

5. Youla, D: Mathematical theory of image restoration by the method of convex projections. In: Stark, H (ed.) Image Recovery Theory and Applications, pp. 29-77. Academic Press, Orlando (1987)

6. Osilike, MO, Isiogugu, FO: Weak and strong convergence theorems for nonspreading-type mappings in Hilbert spaces. Nonlinear Anal., Theory Methods Appl. 74(5), 1814-1822 (2011)

7. Chang, SS, Cho, YJ, Lee, BS, Jung, JS, Kang, SM: Iterative approximations of fixed points and solutions for strongly accretive and strongly pseudo-contractive mappings in Banach spaces. J. Math. Anal. Appl. 224(1), 149-165 (1998)

8. Cho, YJ, Kang, SM, Qin, X: Some results on k-strictly pseudo-contractive mappings in Hilbert spaces. Nonlinear Anal., Theory Methods Appl. 70(5), 1956-1964 (2009)

9. Jung, JS: Strong convergence of iterative methods for $k$-strictly pseudo-contractive mappings in Hilbert spaces. Appl. Math. Comput. 215(10), 3746-3753 (2010)

10. Zhou, HY: Convergence theorems of fixed points for $\kappa$-strict pseudo-contractions in Hilbert spaces. Nonlinear Anal., Theory Methods Appl. 69(2), 456-462 (2008)

11. Wang, S: A general iterative method for obtaining an infinite family of strictly pseudo-contractive mappings in Hilbert spaces. Appl. Math. Lett. 24(6), 901-907 (2011)

12. Petrot, N, Wangkeeree, R: A general iterative scheme for strict pseudononspreading mapping related to optimization problem in Hilbert spaces. J. Nonlinear Anal. Optim., Theory Appl. 2(2), 329-336 (2011)

13. Yao, Y, Liou, YC, Chen, CP: Algorithms construction for nonexpansive mappings and inverse-strongly monotone mappings. Taiwan. J. Math. 15(5), 1979-1998 (2011)

14. Takahashi, W: Strong convergence theorems for maximal and inverse-strongly monotone mappings in Hilbert spaces and applications. J. Optim. Theory Appl. 157(3), 781-802 (2013)

15. Li, L, Song, W: A hybrid of the extragradient method and proximal point algorithm for inverse strongly monotone operators and maximal monotone operators in Banach spaces. Nonlinear Anal. Hybrid Syst. 1(3), 398-413 (2007) 
16. Thianwan, S: Strong convergence theorems by hybrid methods for a finite family of nonexpansive mappings and inverse-strongly monotone mappings. Nonlinear Anal. Hybrid Syst. 3(4), 605-614 (2009)

17. Ke, YF, Ma, CF: A new relaxed extragradient-like algorithm for approaching common solutions of generalized mixed equilibrium problems, a more general system of variational inequalities and a fixed point problem. Fixed Point Theory Appl. 2013, 126 (2013)

18. $\mathrm{Ke}, \mathrm{YF}, \mathrm{Ma}, \mathrm{CF}$ : The convergence analysis of the projection methods for a system of generalized relaxed cocoercive variational inequalities in Hilbert spaces. Fixed Point Theory Appl. 2013, 182 (2013)

19. Ke, YF, Ma, CF: Iterative algorithm of common solutions for a constrained convex minimization problem, a quasi-variational inclusion problem and the fixed point problem of a strictly pseudo-contractive mapping. Fixed Point Theory Appl. 2014, 54 (2014)

20. Takahashi, W, Shimoji, K: Convergence theorems for nonexpansive mappings and feasibility problems. Math. Comput. Model. 32(11), 1463-1471 (2000)

21. Kangtunyakarn, A, Suantai, S: A new mapping for finding common solutions of equilibrium problems and fixed point problems of finite family of nonexpansive mappings. Nonlinear Anal., Theory Methods Appl. 71(10), 4448-4460 (2009)

22. Suwannaut, S, Kangtunyakarn, A: Strong convergence theorem for the modified generalized equilibrium problem and fixed point problem of strictly pseudo-contractive mappings. Fixed Point Theory Appl. 2014, 86 (2014)

23. Kangtunyakarn, A, Suantai, S: Hybrid iterative scheme for generalized equilibrium problems and fixed point problems of finite family of nonexpansive mappings. Nonlinear Anal. Hybrid Syst. 3(3), 296-309 (2009)

24. Kangtunyakarn, A, Suantai, S: Strong convergence of a new iterative scheme for a finite family of strict pseudo-contractions. Comput. Math. Appl. 60(3), 680-694 (2010)

25. Shimoji, K, Takahashi, W: Strong convergence to common fixed points of infinite nonexpansive mappings and applications. Taiwan. J. Math. 5(2), 387-404 (2001)

26. Kikkawa, M, Takahashi, W: Approximating fixed points of infinite nonexpansive mappings by the hybrid method. J. Optim. Theory Appl. 117(1), 93-101 (2003)

27. Nakajo, K, Shimoji, K, Takahashi, W: Strong convergence theorems by the hybrid method for families of nonexpansive mappings in Hilbert spaces. Taiwan. J. Math. 10(2), 339-360 (2006)

28. Ibaraki, T, Takahashi, W: Weak convergence theorem for new nonexpansive mappings in Banach spaces and its applications. Taiwan. J. Math. 11(3), 929-944 (2007)

29. Colao, V, Marino, G, Xu, HK: An iterative method for finding common solutions of equilibrium and fixed point problems. J. Math. Anal. Appl. 344(1), 340-352 (2008)

30. Ceng, LC, Yao, JC: A hybrid iterative scheme for mixed equilibrium problems and fixed point problems. J. Comput. Appl. Math. 214(1), 186-201 (2008)

31. Chang, S, Lee, HWJ, Chan, CK: A new method for solving equilibrium problem fixed point problem and variational inequality problem with application to optimization. Nonlinear Anal., Theory Methods Appl. 70(9), 3307-3319 (2009)

32. Peng, JW, Yao, JC: Strong convergence theorems of iterative scheme based on the extragradient method for mixed equilibrium problems and fixed point problems. Math. Comput. Model. 49(9), 1816-1828 (2009)

33. Piri, $\mathrm{H}$ : A general iterative method for finding common solutions of system of equilibrium problems, system of variational inequalities and fixed point problems. Math. Comput. Model. 55(3), 1622-1638 (2012)

34. Jaiboon, C, Chantarangsi, W, Kumam, P: A convergence theorem based on a hybrid relaxed extragradient method for generalized equilibrium problems and fixed point problems of a finite family of nonexpansive mappings. Nonlinear Anal. Hybrid Syst. 4(1), 199-215 (2010)

35. Kangtunyakarn, A: Convergence theorem of common fixed points for a family of nonspreading mappings in Hilbert space. Optim. Lett. 6(5), 957-961 (2012)

36. Kangtunyakarn, A: Iterative scheme for a nonexpansive mapping, an $\eta$-strictly pseudo-contractive mapping and variational inequality problems in a uniformly convex and 2-uniformly smooth Banach space. Fixed Point Theory Appl. 2013, 23 (2013)

37. Che, HT, Li, MX: A simultaneous iterative method for split equality problems of two finite families of strictly pseudononspreading mappings without prior knowledge of operator norms. Fixed Point Theory Appl. 2015, 1 (2015)

38. Kangtunyakarn, A: Iterative methods for finding common solution of generalized equilibrium problems and variational inequality problems and fixed point problems of a finite family of nonexpansive mappings. Fixed Point Theory Appl. 2010, 836714 (2010)

39. Kangtunyakarn, A: Strong convergence theorem for a generalized equilibrium problem and system of variational inequalities problem and infinite family of strict pseudo-contractions. Fixed Point Theory Appl. 2011, 23 (2011)

40. Kangtunyakarn, A: Iterative algorithms for finding a common solution of system of the set of variational inclusion problems and the set of fixed point problems. Fixed Point Theory Appl. 2011, 38 (2011)

41. Kangtunyakarn, $A$ : A new iterative scheme for fixed point problems of infinite family of $\boldsymbol{\kappa}_{i}$-pseudo contractive mappings, equilibrium problem, variational inequality problems. J. Glob. Optim. 56(4), 1543-1562 (2013)

42. Kangtunyakarn, A: An iterative algorithm to approximate a common element of the set of common fixed points for a finite family of strict pseudo-contractions and of the set of solutions for a modified system of variational inequalities. Fixed Point Theory Appl. 2013, 143 (2013)

43. Kangtunyakarn, A: A new mapping for finding a common element of the sets of fixed points of two finite families of nonexpansive and strictly pseudo-contractive mappings and two sets of variational inequalities in uniformly convex and 2-smooth Banach spaces. Fixed Point Theory Appl. 2013, 157 (2013)

44. Kangtunyakarn, A: The methods for variational inequality problems and fixed point of $\kappa$-strictly pseudononspreading mapping. Fixed Point Theory Appl. 2013, 171 (2013)

45. Xu, HK: An iterative approach to quadratic optimization. J. Optim. Theory Appl. 116(3), 659-678 (2003)

46. Takahashi, W: Nonlinear Functional Analysis: Fixed Point Theory and Its Applications. Yokohama Publishers, Yokohama (2000)

47. Opial, Z: Weak convergence of the sequence of successive approximations for nonexpansive mappings. Bull. Am. Math. Soc. 73(4), 591-597 (1967)

48. Cai, G, Bu, SQ: Hybrid algorithm for generalized mixed equilibrium problems and variational inequality problems and fixed point problems. Comput. Math. Appl. 62, 4772-4782 (2011) 